Pacific

Journal of

Mathematics

COMMUTATION RELATIONS

FOR ARBITRARY QUANTUM MINORS

KENNETH R. GOODEARL 


\title{
COMMUTATION RELATIONS FOR ARBITRARY QUANTUM MINORS
}

\author{
KEnNETH R. GOODEARL
}

\begin{abstract}
Complete sets of commutation relations for arbitrary pairs of quantum minors are computed, with explicit coefficients in closed form.
\end{abstract}

\section{Introduction}

The title of this paper begins with what may seem to be a misnomer; the term commutation relation, in current usage, does not refer to a commutativity condition $x y=y x$, but has evolved to encompass various "skew commutativity" conditions that have proved to be useful replacements for commutativity. Older types of commutation relations include conditions of the form $x y-y x=z$, used in defining Weyl algebras and enveloping algebras. In quantized versions of classical algebras appear relations such as $x y=q y x$ (known as $q$-commutation), along with mixtures of both types. Thus, it has become common to refer to any equation of the form $x y=\lambda y x+z$, where $\lambda$ is a nonzero scalar, as a commutation relation for $x$ and $y$. One important use of such a relation, especially in enveloping algebras, is that, if the algebra supports a filtration such that $\operatorname{deg}(z)<\operatorname{deg}(x)+\operatorname{deg}(y)$, then the images of $x$ and $y$ in the associated graded algebra - call them $\tilde{x}$ and $\tilde{y}$ - commute up to a scalar: $\tilde{x} \tilde{y}=\lambda \tilde{y} \tilde{x}$. Similarly, the cosets of $x$ and $y$ modulo the ideal generated by $z$ commute up to $\lambda$. Such coset relations are key ingredients in the work on quantized coordinate rings of Soibelman [1990], Hodges and Levasseur [1993; 1994], Joseph [1995], and others.

In many quantized algebras, the available commutation relations are homogeneous and quadratic, of the form $x y=\lambda y x+\sum_{i} \mu_{i} x_{i} y_{i}$ (where $\lambda$ and the $\mu_{i}$ are nonzero scalars). Relations of this type are particularly important in establishing a (noncommutative) standard basis of monomials in generators that include the elements $x, y, x_{i}, y_{i}$. Namely, if the generators are ordered in such a way that each $x_{i} \leq y_{i}$ but $x>y$, then the given relation allows one to rewrite monomials involving $x y$ as linear combinations of monomials closer to standard form. For example, noncommutative standard bases have been constructed by Lakshmibai

MSC2000: 16W35, 20G42.

Keywords: quantum matrices, quantum minors, commutation, quasicommutation, $q$-commutation. This research was partially supported by a grant from the National Science Foundation. 
and Reshetikhin [1991; 1992] (for quantized coordinate rings of flag varieties and Schubert schemes), by Goodearl and Lenagan [2000] (for quantum matrix algebras), and by Lenagan and Rigal [2006] (for quantum Grassmannians and quantum determinantal rings).

In order to work effectively with quantized coordinate rings of matrices, Grassmannians, special or general linear groups, and related algebras, one needs explicit commutation relations for quantum minors and related elements. Such relations have often been derived for special cases as needed, either by induction on the size of the minors, using quantum Laplace relations as in Parshall and Wang [1991] and Taft and Towber [1991], or by applying the quasitriangular structure of $U_{q}\left(\mathfrak{s l}_{n}(k)\right)$ (that is, its universal $R$-matrix) to coordinate functions in $O_{q}\left(S L_{n}(k)\right)$, as in the work of Lakshmibai and Reshetikhin [1991; 1992], Soibelman [1990], and Hodges and Levasseur [1993; 1994]. Along the former line, the most complete results to date were obtained by Fioresi [1999; 2004], who developed an algorithm that yields a commutation relation for any pair of quantum minors. This algorithm is an iterative procedure, in which certain products of quantum minors may appear multiple times; explicit coefficients are produced, but are not expressed as closed formulas. Via the quasitriangular approach, general commutation relations for pairs of coordinate functions in quantized coordinate rings $\mathcal{O}_{q}(G)$, where $G$ is a semisimple Lie group, have been derived in special cases (for example, see [Lakshmibai and Reshetikhin 1991; 1992; Soibelman 1990; Hodges and Levasseur $1993 ; 1994]$ ), not all with explicit coefficients. (Quantum minors in $O_{q}\left(S L_{n}(k)\right.$ ) are special coordinate functions.) Perhaps the largest group of explicit commutation relations obtained in this way appeared in Hodges et al. [1997] (see also [Brown and Goodearl 2002]). However, to make these fully explicit, canonical elements for the Rosso-Tanisaki Killing form on $U_{q}\left(\mathfrak{s l}_{n}(k)\right)$ had to be computed.

Here we introduce a new method - new only in the sense that it has apparently not been used for this purpose before - with which we derive complete commutation relations for arbitrary pairs of quantum minors, with explicit coefficients in closed form. Our method is dual to the quasitriangular approach, as it relies on the coquasitriangular (or braided) bialgebra structure on the quantized coordinate ring of $n \times n$ matrices. Representation-theoretically, the two approaches are based on equivalent information, in that a quasitriangular (respectively, coquasitriangular) structure on a bialgebra encodes braiding isomorphisms

$$
V \otimes W \stackrel{\cong}{\longrightarrow} W \otimes V
$$

for finite dimensional modules (respectively, comodules) $V$ and $W$. To record such isomorphisms, one typically requires formulas for matrix entries. However, in the case of a coquasitriangular bialgebra $A$, the above isomorphism information is stored more compactly, in a bilinear form $\mathbf{r}$ on $A$. The braiding isomorphism for 
left $A$-comodules $V$ and $W$ is then given by the formula

$$
v \otimes w \longmapsto \sum_{(v),(w)} \mathbf{r}\left(v_{0}, w_{0}\right) w_{1} \otimes v_{1},
$$

where we have used Sweedler's notation: $v \mapsto \sum_{(v)} v_{0} \otimes v_{1}$ for the comodule structure map $V \rightarrow A \otimes V$, and similarly for $W$. The resulting commutation relations are equations with values of $\mathbf{r}$ as coefficients, namely,

$$
\sum_{(a),(b)} \mathbf{r}\left(a_{1}, b_{1}\right) a_{2} b_{2}=\sum_{(a),(b)} \mathbf{r}\left(a_{2}, b_{2}\right) b_{1} a_{1}
$$

for $a, b \in A$, using now Sweedler's notation for the comultiplication map $A \rightarrow$ $A \otimes A$.

When $A$ is the bialgebra $O_{q}\left(M_{n}(k)\right)$, and $a=[I \mid J]$ and $b=[M \mid N]$ are quantum minors (see below for the notation), equation (1-1) becomes

$$
\sum_{\substack{|S|=|I| \\|T|=|M|}} \mathbf{r}([I \mid S],[M \mid T])[S \mid J][T \mid N]=\sum_{\substack{|S|=|J| \\|T|=|N|}} \mathbf{r}([S \mid J],[T \mid N])[M \mid T][I \mid S] .
$$

Observe that $[I \mid J][M \mid N]$ occurs on the left hand side of (1-2) when $S=I$ and $T=M$, while $[M \mid N][I \mid J]$ occurs on the right when $S=J$ and $T=N$. As we shall see, the coefficients for these terms - namely, $\mathbf{r}([I \mid I],[M \mid M])$ and $\mathbf{r}([J \mid J],[N \mid N])$ - are nonzero (in fact, they are powers of $q$ ). Thus, to obtain explicit commutation relations for $[I \mid J]$ and $[M \mid N]$, we only need to compute the values $\mathbf{r}([I \mid S],[M \mid T])$ and $\mathbf{r}([S \mid J],[T \mid N])$. This is precisely what we do in this paper, see especially Theorems 5.6 and 6.2. Additional relations follow from these by various symmetries, or by using quantum Laplace relations. (Quantum Plücker relations in quantum Grassmannians can also be used for this purpose.) See Theorems 6.7 and 7.3, and Corollaries 6.3, 6.8 and 7.5.

Our notation and conventions are collected in Section 2. In particular, the relations we use for $\mathrm{O}_{q}\left(M_{n}(k)\right)$ are displayed in (2-6), so that the reader may compare them with other papers in which $q$ is replaced by $q^{-1}$ or $q^{2}$. Our computations of the values of the form $\mathbf{r}$ on pairs of quantum minors occupy Sections 3 and 5; the intermediate Section 4 provides a first set of commutation relations to illustrate our methods. The general commutation relations are derived in Sections 6 and 7, and we conclude, in Section 8, by using these relations to evaluate the standard Poisson bracket on pairs of classical minors.

\section{Notation and conventions}

Fix a positive integer $n$, a base field $k$, and a nonzero scalar $q \in k^{\times}$. We work within the standard single-parameter quantized coordinate ring of $n \times n$ matrices 
over $k$, which we denote $\mathrm{O}_{q}\left(M_{n}(k)\right)$, as defined in Section 2.2 below. We use the abbreviation

$$
\hat{q}=q-q^{-1},
$$

since this scalar appears in numerous formulas.

2.1. $R$-matrix. The standard $R$-matrix of type $A_{n-1}$ can be presented in the form

$$
R=q \sum_{i=1}^{n} e_{i i} \otimes e_{i i}+\sum_{\substack{i, j=1 \\ i \neq j}}^{n} e_{i i} \otimes e_{j j}+\hat{q} \sum_{\substack{i, j=1 \\ i>j}}^{n} e_{i j} \otimes e_{j i}
$$

see [Reshetikhin et al. 1989, Equation 1.5, p. 200]. We view $R$ as a linear automorphism of $k^{n} \otimes k^{n}$, which acts on the standard basis vectors $x_{i} \otimes x_{j}$ according to the formula

$$
R\left(x_{l} \otimes x_{m}\right)=\sum_{i, j=1}^{n} R_{l m}^{i j} x_{i} \otimes x_{j},
$$

using the conventions of [Klimyk and Schmüdgen 1997]. The entries of the $n^{2} \times n^{2}$ matrix $R_{l m}^{i j}$ are

$$
\begin{aligned}
& R_{i i}^{i i}=q \quad \text { for all } i, \quad R_{i j}^{i j}=1 \quad \text { for } i \neq j, \\
& R_{j i}^{i j}=\hat{q} \quad \text { for } i>j, \quad R_{l m}^{i j}=0 \quad \text { otherwise; }
\end{aligned}
$$

see [Klimyk and Schmüdgen 1997, Equation 9.13, p. 309].

2.2. Generators, relations, and grading. The algebra $A=O_{q}\left(M_{n}(k)\right)$ is obtained from (2-4) by the Faddeev-Reshetikhin-Takhtadzhyan construction, that is, as the $k$-algebra $A(R)$ presented by generators $X_{i j}$ (for $i, j=1, \ldots, n$ ) and relations

$$
\sum_{s, t=1}^{n} R_{s t}^{i j} X_{s l} X_{t m}=\sum_{s, t=1}^{n} X_{j t} X_{i s} R_{l m}^{s t}
$$

for all $i, j, l, m=1, \ldots, n$ (see [Reshetikhin et al. 1989, Definition 1, p. 197] and [Klimyk and Schmüdgen 1997, Section 9.1.1]; we have written $X_{i j}$ for the generators labelled $t_{i j}$ in [Reshetikhin et al. 1989] and $u_{j}^{i}$ in [Klimyk and Schmüdgen 1997]). As is well known, the relations (2-5) are equivalent to

$$
\begin{aligned}
X_{i j} X_{l j} & =q X_{l j} X_{i j} & & \text { for } i<l, \\
X_{i j} X_{i m} & =q X_{i m} X_{i j} & & \text { for } j<m, \\
X_{i j} X_{l m} & =X_{l m} X_{i j} & & \text { for } i<l \text { and } j>m, \\
X_{i j} X_{l m}-X_{l m} X_{i j} & =\hat{q} X_{i m} X_{l j} & & \text { for } i<l \text { and } j<m
\end{aligned}
$$


(see [Klimyk and Schmüdgen 1997, Equations 9.17, p. 310]). Some authors define quantum matrices using relations as in (2-6) but with $q$ replaced by $q^{-1}$; thus, the algebras they define match what we would label $\mathrm{O}_{q^{-1}}\left(M_{n}(k)\right)$. See, for example, [Larson and Towber 1991, p. 3317] or [Parshall and Wang 1991, Equation 3.5a, p. 37]. In comparing our work with those papers, we must be careful to interchange $q$ and $q^{-1}$; however, $\hat{q}$ is defined to be $q^{-1}-q$ in [Parshall and Wang 1991, p. 38], and so we do not change $\hat{q}$ when carrying over results from that paper.

Because of the homogeneity of the relations (2-6), $A$ carries a natural $\left(\mathbb{Z}^{n} \times \mathbb{Z}^{n}\right)$ grading, such that each $X_{i j}$ is homogeneous of degree $\left(\epsilon_{i}, \epsilon_{j}\right)$, where $\epsilon_{1}, \ldots, \epsilon_{n}$ are the standard basis elements for $\mathbb{Z}^{n}$.

2.3. Coquasitriangular structure. We follow [Hayashi 1992, Section 1] in defining a coquasitriangular bialgebra (also called a bialgebra with braiding structure [Larson and Towber 1991, Theorem 2.7] or a cobraided bialgebra [Kassel 1995, Definition VIII.5.1]) to be a bialgebra $B$ equipped with a convolution-invertible bilinear form $\mathbf{r}: B \otimes B \rightarrow k$ such that

$$
\sum_{(a),(b)} \mathbf{r}\left(a_{1}, b_{1}\right) a_{2} b_{2}=\sum_{(a),(b)} \mathbf{r}\left(a_{2}, b_{2}\right) b_{1} a_{1},
$$

$$
\begin{aligned}
& \mathbf{r}(a b, c)=\sum_{(c)} \mathbf{r}\left(a, c_{1}\right) \mathbf{r}\left(b, c_{2}\right), \\
& \mathbf{r}(a, b c)=\sum_{(a)} \mathbf{r}\left(a_{1}, c\right) \mathbf{r}\left(a_{2}, b\right), \\
& \mathbf{r}(a, 1)=\mathbf{r}(1, a)=\varepsilon(a)
\end{aligned}
$$

for all $a, b, c \in B$, where $\mathbf{r}(x, y)$ stands for $\mathbf{r}(x \otimes y)$ for convenience and we use Sweedler's notation for comultiplication, in the form $\Delta(x)=\sum_{(x)} x_{1} \otimes x_{2}$. Condition (2-10) is redundant by [Klimyk and Schmüdgen 1997, Proposition 10.2(ii), p. 333]. Thus, the above definition agrees with [Kassel 1995, Definition VIII.5.1], [Klimyk and Schmüdgen 1997, Definition 10.1, pp. 331-2], and [Lambe and Radford 1997, Definition 7.3.1], but not with the conditions in [Larson and Towber 1991, Theorem 2.7]. However, the latter conditions match those of (2-10) if one uses the form $\langle\cdot \mid \cdot\rangle$ given by $\langle a \mid b\rangle=\mathbf{r}(b, a)$.

By [Klimyk and Schmüdgen 1997, Theorem 10.7, p. 337], whenever $R$ is an invertible $R$-matrix satisfying the original form of the quantum Yang-Baxter equation, the FRT-algebra $A(R)$ is coquasitriangular with respect to the form $\mathbf{r}$ determined by

$$
\mathbf{r}\left(X_{i j}, X_{l m}\right)=R_{j m}^{i l}
$$


for all $i, j, l, m$. (By "original quantum Yang-Baxter equation" we mean the equation $R_{12} R_{13} R_{23}=R_{23} R_{13} R_{12}$ [Reshetikhin et al. 1989, Equation 0.7, p. 195], as opposed to the form exhibiting the braid relation, namely $R_{12} R_{23} R_{12}=R_{23} R_{12} R_{23}$.) Note that, in view of (2-11), if we put $a=X_{i l}$ and $b=X_{j m}$ into (2-7), we recover relations (2-5).

It is well known that the $R$-matrix given in (2-2) satisfies the original quantum Yang-Baxter equation (see, for example, [Klimyk and Schmüdgen 1997, Section 8.1.2, pp. 246-7, and Equation 8.60, p. 270]). Consequently:

Theorem 2.4. The algebra $A=\mathrm{O}_{q}\left(M_{n}(k)\right)$ is a coquasitriangular bialgebra with respect to the bilinear form $\mathbf{r}: A \otimes A \rightarrow k$ determined by the following conditions:

$$
\begin{array}{llll}
\mathbf{r}\left(X_{i i}, X_{i i}\right)=q & \text { for all } i, & \mathbf{r}\left(X_{i i}, X_{j j}\right)=1 & \text { for } i \neq j, \\
\mathbf{r}\left(X_{i j}, X_{j i}\right)=\hat{q} & \text { for } i>j, & \mathbf{r}\left(X_{i j}, X_{l m}\right)=0 & \text { otherwise. }
\end{array}
$$

2.5. Quantum minors. We write $[I \mid J]$ for the quantum minor in $A$ with rowindex set $I$ and column-index set $J$; this minor is just the quantum determinant in the subalgebra $k\left\langle X_{i j} \mid i \in I, j \in J\right\rangle$, which is naturally isomorphic to $O_{q}\left(M_{|I|}(k)\right)$. Specifically, if we write the elements of $I$ and $J$ in ascending order, say,

$$
I=\left\{i_{1}<\cdots<i_{t}\right\} \quad \text { and } \quad J=\left\{j_{1}<\cdots<j_{t}\right\}
$$

then

$$
\begin{aligned}
{[I \mid J] } & =\sum_{\sigma \in S_{t}}(-q)^{\ell(\sigma)} X_{i_{\sigma(1)}, j_{1}} X_{i_{\sigma(2)}, j_{2}} \cdots X_{i_{\sigma(t)}, j_{t}} \\
& =\sum_{\sigma \in S_{t}}(-q)^{\ell(\sigma)} X_{i_{1}, j_{\sigma(1)}} X_{i_{2}, j_{\sigma(2)}} \cdots X_{i_{t}, j_{\sigma(t)}},
\end{aligned}
$$

where $\ell(\sigma)$ denotes the length of the permutation $\sigma \in S_{t}$ as a product of simple transpositions $(l, l+1)$ (see [Klimyk and Schmüdgen 1997, Equations 9.18 and 9.20, pp. 311-312], [Parshall and Wang 1991, p. 43]). Note that $[I \mid J]$ is homogeneous of degree

$$
\left(\epsilon_{i_{1}}+\cdots+\epsilon_{i_{t}}, \epsilon_{j_{1}}+\cdots+\epsilon_{j_{t}}\right)
$$

with respect to the grading of Section 2.2.

Comultiplication of quantum minors is given by the rule

$$
\Delta([I \mid J])=\sum_{\substack{K \subseteq\{1, \ldots, n\} \\|K|=|I|}}[I \mid K][K \mid J]
$$

(see, for example, [Klimyk and Schmüdgen 1997, Proposition 9.7(ii), p. 312]). 
2.6. Transpose and antitranspose. As observed in [Parshall and Wang 1991, Proposition 3.7.1(1)], there is a $k$-algebra automorphism $\tau$ on $A$ such that $\tau\left(X_{i j}\right)=X_{j i}$ for all $i, j$. We refer to $\tau$ as the transpose automorphism. There is also a $k$-algebra anti-automorphism $\tau_{2}$ on $A$ sending $X_{i j} \mapsto X_{n+1-i, n+1-j}$ for all $i, j$ [Parshall and Wang 1991, Proposition 3.7.1(2)]. This proposition also shows that $\tau$ is a coalgebra anti-automorphism, while $\tau_{2}$ is a coalgebra automorphism; that is,

$$
\Delta \circ \tau=\phi \circ(\tau \otimes \tau) \circ \Delta \quad \text { and } \quad \Delta \circ \tau_{2}=\left(\tau_{2} \otimes \tau_{2}\right) \circ \Delta,
$$

where $\phi$ is the flip automorphism on $A \otimes A$, sending $a \otimes b \mapsto b \otimes a$ for all $a, b \in A$. Hence,

$$
\Delta \tau(a)=\sum_{(a)} \tau\left(a_{2}\right) \otimes \tau\left(a_{1}\right) \quad \text { and } \quad \Delta \tau_{2}(a)=\sum_{(a)} \tau_{2}\left(a_{1}\right) \otimes \tau_{2}\left(a_{2}\right)
$$

for $a \in A$. Consequently, when writing out $\Delta \tau(a)$ and $\Delta \tau_{2}(a)$ in Sweedler's notation, we may take

$$
\begin{aligned}
\tau(a)_{1} & =\tau\left(a_{2}\right), & \tau(a)_{2} & =\tau\left(a_{1}\right), \\
\tau_{2}(a)_{1} & =\tau_{2}\left(a_{1}\right), & \tau_{2}(a)_{2} & =\tau_{2}\left(a_{2}\right) .
\end{aligned}
$$

We recall from [Parshall and Wang 1991, Lemma 4.3.1] that

$$
\tau([I \mid J])=[J \mid I] \quad \text { and } \quad \tau_{2}([I \mid J])=\left[\omega_{0} I \mid \omega_{0} J\right]
$$

for all quantum minors $[I \mid J]$ in $A$, where $\omega_{0}$ is the longest element of $S_{n}$, that is, the permutation $i \mapsto n+1-i$.

As discussed in [Parshall and Wang 1991, Remark 3.7.2], there is an isomorphism (of bialgebras) $\mathscr{O}_{q}\left(M_{n}(k)\right) \rightarrow \mathcal{O}_{q^{-1}}\left(M_{n}(k)\right)$ that sends $X_{i j} \mapsto X_{n+1-i, n+1-j}^{\prime}$ for all $i, j$, where the $X_{.,}^{\prime}$ are the standard generators for $O_{q^{-1}}\left(M_{n}(k)\right)$. We call this isomorphism $\beta$ and use the notation $[I \mid J]^{\prime}$ for quantum minors in $O_{q^{-1}}\left(M_{n}(k)\right)$. It was shown in [Goodearl and Lenagan 2003, proof of Corollary 5.9] that

$$
\beta([I \mid J])=\left[\omega_{0} I \mid \omega_{0} J\right]^{\prime}
$$

for all quantum minors $[I \mid J]$ in $A$.

Lemma 2.7. The form $\mathbf{r}$ satisfies $\mathbf{r}(a, b)=\mathbf{r}(\tau(b), \tau(a))=\mathbf{r}\left(\tau_{2}(b), \tau_{2}(a)\right)$ for all $a, b \in A$. In particular,

$$
\mathbf{r}([I \mid J],[M \mid N])=\mathbf{r}([N \mid M],[J \mid I])=\mathbf{r}\left(\left[\omega_{0} M \mid \omega_{0} N\right],\left[\omega_{0} I \mid \omega_{0} J\right]\right)
$$

for all quantum minors $[I \mid J]$ and $[M \mid N]$ in $A$.

Proof. Set $\mathbf{r}^{\prime}(a, b)=\mathbf{r}(\tau(b), \tau(a))$ and $\mathbf{r}^{\prime \prime}(a, b)=\mathbf{r}\left(\tau_{2}(b), \tau_{2}(a)\right)$ for all $a, b \in A$. From (2-12), note that $\mathbf{r}^{\prime}\left(X_{i j}, X_{l m}\right)=\mathbf{r}^{\prime \prime}\left(X_{i j}, X_{l m}\right)=\mathbf{r}\left(X_{i j}, X_{l m}\right)$ for all $i, j, l, m$. To prove that $\mathbf{r}^{\prime}$ and $\mathbf{r}^{\prime \prime}$ coincide with $\mathbf{r}$, it suffices to show that these forms agree 
on all monomials in the $X_{i j}$. This will be clear by induction on the lengths of the monomials, once we show that $\mathbf{r}^{\prime}$ and $\mathbf{r}^{\prime \prime}$ satisfy (2-8) and (2-9). With the aid of $(2-15)$, these identities are routine; we give one sample:

$$
\begin{aligned}
\mathbf{r}^{\prime}(a b, c) & =\mathbf{r}(\tau(c), \tau(a) \tau(b)) \\
& =\sum_{(\tau(c))} \mathbf{r}\left(\tau(c)_{1}, \tau(b)\right) \mathbf{r}\left(\tau(c)_{2}, \tau(a)\right)=\sum_{(c)} \mathbf{r}\left(\tau\left(c_{2}\right), \tau(b)\right) \mathbf{r}\left(\tau\left(c_{1}\right), \tau(a)\right) \\
& =\sum_{(c)} \mathbf{r}^{\prime}\left(b, c_{2}\right) \mathbf{r}^{\prime}\left(a, c_{1}\right)=\sum_{(c)} \mathbf{r}^{\prime}\left(a, c_{1}\right) \mathbf{r}^{\prime}\left(b, c_{2}\right),
\end{aligned}
$$

for all $a, b, c \in A$.

2.8. Definition of the quantities $\ell(S ; T)$. Many formulas concerning quantum minors require powers of $q$ or $-q$ whose exponents are quantities that might be called the number of inversions between two sets. We follow [Noumi et al. 1993] in defining

$$
\ell(S ; T)=|\{(s, t) \in S \times T \mid s>t\}|
$$

for any subsets $S, T \subseteq\{1, \ldots, n\}$.

2.9. Quantum Laplace relations. We shall need the following $q$-Laplace relations from [Noumi et al. 1993, Proposition 1.1], for index sets $I, J \subseteq\{1, \ldots, n\}$ of the same cardinality. If $I_{1}, I_{2}$ are nonempty subsets of $I$ with $\left|I_{1}\right|+\left|I_{2}\right|=|I|$, then

$$
\sum_{\substack{J=J_{1} \sqcup J_{2} \\\left|J_{l}\right|=\left|I_{l}\right|}}(-q)^{\ell\left(J_{1} ; J_{2}\right)}\left[I_{1} \mid J_{1}\right]\left[I_{2} \mid J_{2}\right]= \begin{cases}(-q)^{\ell\left(I_{1} ; I_{2}\right)}[I \mid J] & \text { if } I_{1} \cap I_{2}=\varnothing \\ 0 & \text { if } I_{1} \cap I_{2} \neq \varnothing\end{cases}
$$

while if $J_{1}, J_{2}$ are nonempty subsets of $J$ with $\left|J_{1}\right|+\left|J_{2}\right|=|J|$, then

$$
\sum_{\substack{I=I_{1} \cup I_{2} \\\left|I_{l}\right|=\left|J_{l}\right|}}(-q)^{\ell\left(I_{1} ; I_{2}\right)}\left[I_{1} \mid J_{1}\right]\left[I_{2} \mid J_{2}\right]= \begin{cases}(-q)^{\ell\left(J_{1} ; J_{2}\right)}[I \mid J] & \text { if } J_{1} \cap J_{2}=\varnothing \\ 0 & \text { if } J_{1} \cap J_{2} \neq \varnothing\end{cases}
$$

Observe that (2-20) holds trivially in case $I_{1}$ or $I_{2}$ is empty, and that (2-21) holds trivially in case $J_{1}$ or $J_{2}$ is empty

Reduction formulas for the values of the form $\mathbf{r}$ can be obtained by combining (2-8) and (2-9) with (2-20) and (2-21). For example, if $J=J_{1} \sqcup J_{2}$, then (2-21) 
together with (2-8) yields

$$
\begin{aligned}
(-q)^{\ell\left(J_{1} ; J_{2}\right)} & \mathbf{r}([I \mid J],[M \mid N]) \\
& =\sum_{I=I_{1} \sqcup I_{2}} \sum_{L}(-q)^{\ell\left(I_{1} ; I_{2}\right)} \mathbf{r}\left(\left[I_{1} \mid J_{1}\right],[M \mid L]\right) \mathbf{r}\left(\left[I_{2} \mid J_{2}\right],[L \mid N]\right)
\end{aligned}
$$

for all $[M \mid N]$.

2.10. Some further notation. To simplify notation for operations on index sets, we often omit braces from singletons; in particular, we write

$$
I \backslash i=I \backslash\{i\}, \quad I \sqcup l=I \sqcup\{l\}, \quad I \backslash i \sqcup l=(I \backslash\{i\}) \sqcup\{l\},
$$

for $i \in I$ and $l \notin I$. The Kronecker delta symbol will be applied to index sets as well as to individual indices; thus, $\delta(I, J)=1$ when $I=J$, while $\delta(I, J)=0$ when $I \neq J$. In the case of an index versus an index set, the Kronecker symbol will be used to indicate membership, that is, $\delta(i, I)=1$ means $i \in I$, while $\delta(i, I)=0$ means $i \notin I$.

Finally, we shall need the following partial order on index sets of the same cardinality. If $I$ and $J$ are $t$-element subsets of $\{1, \ldots, n\}$, write their elements in ascending order, say,

$$
I=\left\{i_{1}<i_{2}<\cdots<i_{t}\right\} \quad \text { and } \quad J=\left\{j_{1}<j_{2}<\cdots<j_{t}\right\},
$$

and then define

$$
I \leq J \quad \text { if and only if } \quad i_{l} \leq j_{l} \quad \text { for } l=1, \ldots, t .
$$

\section{Initial computations}

Throughout this section, let $i$ and $j$ denote indices in $\{1, \ldots, n\}$, and let $I, J, M$, $N$ denote index sets contained in $\{1, \ldots, n\}$, with $|I|=|J|$ and $|M|=|N|$.

Lemma 3.1. $\mathbf{r}\left(X_{i i},[I \mid J]\right)=\mathbf{r}\left([I \mid J], X_{i i}\right)=q^{\delta(i, I)} \delta(I, J)$.

Proof. Write $I=\left\{i_{1}<\cdots<i_{t}\right\}$ and $J=\left\{j_{1}<\cdots<j_{t}\right\}$, and, using (2-13) and (2-8), note that

$$
\begin{aligned}
& \mathbf{r}\left([I \mid J], X_{i i}\right) \\
& \quad=\sum_{\sigma \in S_{t}}(-q)^{\ell(\sigma)} \sum_{l_{1}, \ldots, l_{t-1}}^{n} \mathbf{r}\left(X_{i_{1} j_{\sigma(1)}}, X_{i l_{1}}\right) \mathbf{r}\left(X_{i_{2} j_{\sigma(2)}}, X_{l_{1} l_{2}}\right) \cdots \mathbf{r}\left(X_{i_{t} j_{\sigma(t)}}, X_{l_{t-1} i}\right) .
\end{aligned}
$$

In view of (2-12), a nonzero term can occur in the second summation of (3-1) only when $i \leq l_{1} \leq l_{2} \leq \cdots \leq l_{t-1} \leq i$, that is, when $l_{1}=\cdots=l_{t-1}=i$. Hence, (3-1) 
reduces to

$$
\begin{aligned}
& \mathbf{r}\left([I \mid J], X_{i i}\right) \\
& =\sum_{\sigma \in S_{t}}(-q)^{\ell(\sigma)} \mathbf{r}\left(X_{i_{1} j_{\sigma(1)}}, X_{i i}\right) \mathbf{r}\left(X_{i_{2} j_{\sigma(2)}}, X_{i i}\right) \cdots \mathbf{r}\left(X_{i_{t} j_{\sigma(t)}}, X_{i i}\right) .
\end{aligned}
$$

In (3-2), a nonzero term can occur in the sum only when $i_{s}=j_{\sigma(s)}$ for $s=1, \ldots, t$. Since the $i_{s}$ and $j_{s}$ are arranged in ascending order, this situation only happens when $I=J$ and $\sigma=\mathrm{id}$. Thus, $\mathbf{r}\left([I \mid J], X_{i i}\right)=0$ when $I \neq J$, and

$$
\mathbf{r}\left([I \mid I], X_{i i}\right)=\mathbf{r}\left(X_{i_{1} i_{1}}, X_{i i}\right) \mathbf{r}\left(X_{i_{2} i_{2}}, X_{i i}\right) \cdots \mathbf{r}\left(X_{i_{t} i_{t}}, X_{i i}\right)=q^{\delta(i, I)} .
$$

The formula for $\mathbf{r}\left(X_{i i},[I \mid J]\right)$ follows via Lemma 2.7.

Lemma 3.2. $\mathbf{r}\left(X_{i j}, \cdot\right) \equiv 0$ when $i<j$, and $\mathbf{r}\left(\cdot, X_{i j}\right) \equiv 0$ when $i>j$.

Proof. Consider any monomial $a=X_{i(1), j(1)} X_{i(2), j(2)} \cdots X_{i(t), j(t)} \in A$. By (2-8),

$$
\mathbf{r}\left(a, X_{i j}\right)=\sum_{l_{1}, \ldots, l_{t-1}}^{n} \mathbf{r}\left(X_{i(1), j(1)}, X_{i l_{1}}\right) \mathbf{r}\left(X_{i(2), j(2)}, X_{l_{1} l_{2}}\right) \cdots \mathbf{r}\left(X_{i(t), j(t)}, X_{l_{t-1} j}\right) .
$$

If some term $\mathbf{r}\left(X_{i(1), j(1)}, X_{i l_{1}}\right) \mathbf{r}\left(X_{i(2), j(2)}, X_{l_{1} l_{2}}\right) \cdots \mathbf{r}\left(X_{i(t), j(t)}, X_{l_{t-1} j}\right)$ does not vanish, we must have $i \leq l_{1} \leq \cdots \leq l_{t-1} \leq j$. This shows that $\mathbf{r}\left(\cdot, X_{i j}\right)$ can fail to vanish only when $i \leq j$. The first statement of the lemma follows via Lemma 2.7 .

Corollary 3.3. $\mathbf{r}([I \mid J], \cdot) \equiv 0$ when $I \nsupseteq J$, and $\mathbf{r}(\cdot,[I \mid J]) \equiv 0$ when $I \not \leq J$.

Proof. Write $I=\left\{i_{1}<\cdots<i_{t}\right\}$ and $J=\left\{j_{1}<\cdots<j_{t}\right\}$, and suppose that $\mathbf{r}([I \mid J], c) \neq 0$ for some $c \in A$. Then, by (2-13) and (2-8),

$$
\sum_{(c)} \mathbf{r}\left(X_{i_{1} j_{\sigma(1)}}, c_{1}\right) \mathbf{r}\left(X_{i_{2} j_{\sigma(2)}}, c_{2}\right) \cdots \mathbf{r}\left(X_{i_{t} j_{\sigma(t)}}, c_{t}\right) \neq 0
$$

for some $\sigma \in S_{t}$. Lemma 3.2 then implies that $i_{s} \geq j_{\sigma(s)}$ for $s=1, \ldots, t$.

First, $i_{1} \geq j_{\sigma(1)} \geq j_{1}$. Now let $1<s \leq t$. If $\sigma(s) \geq s$, then $i_{s} \geq j_{\sigma(s)} \geq j_{s}$. If $\sigma(s)<s$, then $\sigma(u) \geq s$ for some $u<s$, whence $i_{s}>i_{u} \geq j_{\sigma(u)} \geq j_{s}$. Thus, $i_{s} \geq j_{s}$ for all $s$, and therefore $I \geq J$. Similarly, if $\mathbf{r}(\cdot,[I \mid J])$ does not vanish, then $I \leq J$.

Proposition 3.4. If $i<j$, then

$$
\begin{aligned}
\mathbf{r}\left([I \mid J], X_{i j}\right) & =\hat{q}(-q)^{|[1, i) \cap J|-|[1, j) \cap I|} \delta(i, J) \delta(j, I) \delta(I \backslash j, J \backslash i) \\
& =\hat{q}(-q)^{-|(i, j) \cap I \cap J|} \delta(i, J) \delta(j, I) \delta(I \backslash j, J \backslash i) .
\end{aligned}
$$


Proof. Note first that (3-4) follows from (3-3). For, if the right-hand side of (3-3) is nonzero, then $I=(I \cap J) \sqcup j$ and $J=(I \cap J) \sqcup i$, whence $[1, i) \cap J=[1, i) \cap I \cap J=$ $[1, i] \cap I \cap J$ and $[1, j) \cap I=[1, j) \cap I \cap J$.

We induct on $|I|$, the case $|I|=1$ being clear from (2-12). Assume that $|I|>1$, and suppose that $\mathbf{r}\left([I \mid J], X_{i j}\right) \neq 0$.

Choose $s \in I$, and write $I=I_{1} \sqcup I_{2}$ with $I_{1}=\{s\}$ and $I_{2}=I \backslash\{s\}$. The $q$-Laplace relation (2-20) yields

$$
(-q)^{|[1, s) \cap I|}[I \mid J]=\sum_{t \in J}(-q)^{|[1, t) \cap J|} X_{s t}[I \backslash s \mid J \backslash t] .
$$

For each $t \in J$, we have

$$
\mathbf{r}\left(X_{s t}[I \backslash s \mid J \backslash t], X_{i j}\right)=\sum_{l=1}^{n} \mathbf{r}\left(X_{s t}, X_{i l}\right) \mathbf{r}\left([I \backslash s \mid J \backslash t], X_{l j}\right) .
$$

Since $\mathbf{r}\left([I \mid J], X_{i j}\right) \neq 0$, we must have $\mathbf{r}\left(X_{s t}, X_{i l}\right) \mathbf{r}\left([I \backslash s \mid J \backslash t], X_{l j}\right) \neq 0$ for some $l \in\{1, \ldots, n\}$ and $t \in J$.

Suppose that $i \notin J$. Then $t \neq i$, and so, because $\mathbf{r}\left(X_{s t}, X_{i l}\right) \neq 0$, we must have $t=s$ and $l=i$. Then $\mathbf{r}\left([I \backslash s \mid J \backslash s], X_{i j}\right) \neq 0$, which contradicts the induction hypothesis because $i \notin J \backslash s$. Therefore $i \in J$.

Next, suppose that $j \notin I \backslash s$. If $l<j$, we would have $\mathbf{r}\left([I \backslash s \mid J \backslash t], X_{l j}\right)=0$ by the induction hypothesis. Since $\mathbf{r}\left(\cdot, X_{l j}\right)$ would vanish if $l>j$, we must have $l=j$. Now $\mathbf{r}\left(X_{s t}, X_{i j}\right) \neq 0$, and so $s=j$ and $t=i$. Thus, either $j \in I \backslash s$ or $j=s$, and so in any case we conclude that $j \in I$.

We may now assume that $s=j$. Since $j \notin I \backslash j$, we have $\mathbf{r}\left([I \backslash j \mid J \backslash t], X_{i j}\right)=0$ for all $t \in J$ by the induction hypothesis. On the other hand, $\mathbf{r}\left(X_{j t}, X_{i l}\right)=0$ for $l \neq i, j$, and $\mathbf{r}\left(X_{j t}, X_{i j}\right)=0$ for $t \neq i$. Hence, the right-hand side of (3-6) vanishes when $t \neq i$, and it equals $\hat{q} \mathbf{r}\left([I \backslash j \mid J \backslash i], X_{j j}\right)$ when $t=i$. Combining (3-5) and (3-6) thus yields

$$
(-q)^{|[1, j) \cap I|} \mathbf{r}\left([I \mid J], X_{i j}\right)=(-q)^{|[1, i) \cap J|} \hat{q} \mathbf{r}\left([I \backslash j \mid J \backslash i], X_{j j}\right) .
$$

Since the left-hand side of (3-7) is nonzero by assumption, Lemma 3.1 implies that $I \backslash j=J \backslash i$ and $\mathbf{r}\left([I \backslash j \mid J \backslash i], X_{j j}\right)=1$. The formula (3-3) follows, and the induction step is established.

Corollary 3.5. If $i>j$, then

$$
\begin{aligned}
\mathbf{r}\left(X_{i j},[I \mid J]\right) & =\hat{q}(-q)^{|[1, j) \cap I|-|[1, i) \cap J|} \delta(i, J) \delta(j, I) \delta(I \backslash j, J \backslash i) \\
& =\hat{q}(-q)^{-|(j, i) \cap I \cap J|} \delta(i, J) \delta(j, I) \delta(I \backslash j, J \backslash i) .
\end{aligned}
$$

Proof. Apply Lemma 2.7 to Proposition 3.4.

Proposition 3.6. $\mathbf{r}([I \mid I],[M \mid N])=\mathbf{r}([M \mid N],[I \mid I])=q^{|I \cap M|} \delta(M, N)$. 
Proof. This is parallel to the proof of Lemma 3.1. Write $M=\left\{m_{1}<\cdots<m_{t}\right\}$ and $N=\left\{n_{1}<\cdots<n_{t}\right\}$, and note that

$$
\mathbf{r}([M \mid N],[I \mid I])=\sum_{\sigma \in S_{t}}(-q)^{\ell(\sigma)} \mathbf{r}\left(X_{m_{1} n_{\sigma(1)}} X_{m_{2} n_{\sigma(2)}} \cdots X_{m_{t} n_{\sigma(t)}},[I \mid I]\right),
$$

while for each $\sigma \in S_{t}$ we have

$$
\begin{aligned}
& \mathbf{r}\left(X_{m_{1} n_{\sigma(1)}} X_{m_{2} n_{\sigma(2)}} \cdots X_{m_{t} n_{\sigma(t)}},[I \mid I]\right) \\
= & \sum_{L_{1}, \ldots, L_{t-1}} \mathbf{r}\left(X_{m_{1} n_{\sigma(1)}},\left[I \mid L_{1}\right]\right) \mathbf{r}\left(X_{m_{2} n_{\sigma(2)}},\left[L_{1} \mid L_{2}\right]\right) \cdots \mathbf{r}\left(X_{m_{t} n_{\sigma(t)}},\left[L_{t-1} \mid I\right]\right) .
\end{aligned}
$$

Consider the right-hand side of (3-11). By Corollary 3.3, a nonzero term can occur in that sum only when $I \leq L_{1} \leq \cdots \leq L_{t-1} \leq I$, and so only when all $L_{s}=I$. Thus,

$$
\begin{aligned}
& \mathbf{r}([M \mid N],[I \mid I]) \\
& =\sum_{\sigma \in S_{t}}(-q)^{\ell(\sigma)} \mathbf{r}\left(X_{m_{1} n_{\sigma(1)}},[I \mid I]\right) \mathbf{r}\left(X_{m_{2} n_{\sigma(2)}},[I \mid I]\right) \cdots \mathbf{r}\left(X_{m_{t} n_{\sigma(t)}},[I \mid I]\right)
\end{aligned}
$$

In view of Lemma 3.2 and Corollary 3.5, $\mathbf{r}\left(X_{i j},[I \mid I]\right)=0$ for all $i \neq j$. Hence, a nonzero term can occur in the right-hand side of (3-12) only when $m_{s}=n_{\sigma(s)}$ for all $s$, that is, only when $M=N$ and $\sigma=\mathrm{id}$. Therefore, $\mathbf{r}([M \mid N],[I \mid I])=0$ when $M \neq N$, while, in view of Lemma 3.1,

$\mathbf{r}([M \mid M],[I \mid I])=\mathbf{r}\left(X_{m_{1} m_{1}},[I \mid I]\right) \mathbf{r}\left(X_{m_{2} m_{2}},[I \mid I]\right) \cdots \mathbf{r}\left(X_{m_{t} m_{t}},[I \mid I]\right)=q^{|I \cap M|}$.

The formula for $\mathbf{r}([I \mid I],[M \mid N])$ follows via Lemma 2.7 .

\section{Initial commutation relations}

We now use the computations of $\mathbf{r}(\cdot, \cdot)$ obtained so far to derive some commutation relations, both to illustrate the method and to double-check the results against known relations in the literature. As in the previous section, let $i$ and $j$ denote indices in $\{1, \ldots, n\}$, and let $I, J, M, N$ denote index sets contained in $\{1, \ldots, n\}$, with $|I|=|J|$ and $|M|=|N|$.

4.1. Direct application of (2-7). If we set $a=X_{i j}$ and $b=[I \mid J]$ in (2-7), we obtain

$$
\sum_{l, L} \mathbf{r}\left(X_{i l},[I \mid L]\right) X_{l j}[L \mid J]=\sum_{l, L} \mathbf{r}\left(X_{l j},[L \mid J]\right)[I \mid L] X_{i l}
$$


We claim that (4-1) reduces to

$$
\begin{aligned}
& q^{\delta(i, I)} X_{i j}[I \mid J]+(1-\delta(i, I)) \hat{q} \sum_{\substack{l \in I \\
l<i}}(-q)^{-|(l, i) \cap I|} X_{l j}[I \backslash l \sqcup i \mid J] \\
& \quad=q^{\delta(j, J)}[I \mid J] X_{i j}+(1-\delta(j, J)) \hat{q} \sum_{\substack{l \in J \\
l>j}}(-q)^{-|(j, l) \cap J|}[I \mid J \backslash l \sqcup j] X_{i l} .
\end{aligned}
$$

According to Lemma 3.2 and Corollary 3.3, $\mathbf{r}\left(X_{i l},[I \mid L]\right)=0$ unless $i \geq l$ and $I \leq L$. By Lemma 3.1, $\mathbf{r}\left(X_{i i},[I \mid L]\right)=0$ unless $L=I$, and $\mathbf{r}\left(X_{i i},[I \mid I]\right)=q^{\delta(i, I)}$. When $i>l$, Corollary 3.5 shows that $\mathbf{r}\left(X_{i l},[I \mid L]\right)$ is nonzero only when $i \in L$, $l \in I$, and $I \backslash l=L \backslash i$. In such cases, $i \notin I$ and $L=I \backslash l \sqcup i$, and the exponent of $-q$ that appears in (3-9) is $-|(l, i) \cap I \cap L|=-|(l, i) \cap I|$. Thus, the left-hand sides of (4-1) and (4-2) agree.

Similarly, $\mathbf{r}\left(X_{l j},[L \mid J]\right)=0$ unless $l \geq j$ and $L \leq J$, while $\mathbf{r}\left(X_{j j},[L \mid J]\right)=0$ unless $L=J$, and $\mathbf{r}\left(X_{j j},[J \mid J]\right)=q^{\delta(j, J)}$. When $l>j$, Corollary 3.5 shows that $\mathbf{r}\left(X_{l j},[L \mid J]\right)$ is nonzero only when $l \in J, j \in L \backslash J$, and $L=J \backslash l \sqcup j$. In such cases, the exponent of $-q$ that appears in (3-9) is $-|(j, l) \cap L \cap J|=-|(j, l) \cap J|$. Therefore, the right-hand sides of (4-1) and (4-2) agree. This establishes (4-2).

4.2. Application of the transpose automorphism. There are several ways to obtain a second commutation relation of a kind similar to (4-2). First, we could set $a=[I \mid J]$ and $b=X_{i j}$ in (2-7) and proceed as above. Alternatively, we could apply the automorphism $\tau$, the anti-automorphism $\tau_{2}$, or the isomorphism $\beta$ of Section 2.6 to (4-2) itself. As we shall see in Remark 4.4, the first three ways are equivalent, up to some relabelling. The use of $\beta$ is discussed in Section 4.5.

Among the first three alternatives above, the most convenient choice is to apply the transpose automorphism $\tau$ to (4-2). If we do this, and then relabel the terms by interchanging $i \leftrightarrow j$ and $I \leftrightarrow J$, we obtain

$$
\begin{aligned}
q^{\delta(j, J)} & X_{i j}[I \mid J]+(1-\delta(j, J)) \hat{q} \sum_{\substack{l \in J \\
l<j}}(-q)^{-|(l, j) \cap J|} X_{i l}[I \mid J \backslash l \sqcup j] \\
= & q^{\delta(i, I)}[I \mid J] X_{i j}+(1-\delta(i, I)) \hat{q} \sum_{\substack{l \in I \\
l>i}}(-q)^{-|(i, l) \cap I|}[I \backslash l \sqcup i \mid J] X_{l j} .
\end{aligned}
$$

4.3. Some known cases. We now compare some cases of (4-2) and (4-3) with the literature.

When $i \in I$ and $j \in J$, (4-2) and (4-3) both yield $q X_{i j}[I \mid J]=q[I \mid J] X_{i j}$ - the well-known fact that $X_{i j}$ and $[I \mid J]$ commute in that case (this is just the centrality of the quantum determinant in the subalgebra $k\left\langle X_{s t} \mid s \in I, t \in J\right\rangle$ ). If $i \in I$ and 
$j \notin J$, then (4-2) yields

$$
q X_{i j}[I \mid J]=[I \mid J] X_{i j}+\hat{q} \sum_{\substack{l \in J \\ l>j}}(-q)^{-|(j, l) \cap J|}[I \mid J \backslash l \sqcup j] X_{i l} .
$$

Multiply (4-4) by $q^{-1}$, and note that

$$
q^{-1}(-q)^{-|(j, l) \cap J|}=-(-q)^{-|[j, l] \cap J|} .
$$

Thus modified, (4-4) recovers [Goodearl and Lenagan 2000, Lemma A.1(b)] (this is the second equation of [Parshall and Wang 1991, Lemma 4.5.1(2)], rewritten using the present notation). Similarly, consider the case that $i \notin I$ and $j \in J$. Then (4-3) yields

$$
q X_{i j}[I \mid J]=[I \mid J] X_{i j}+\hat{q} \sum_{\substack{l \in I \\ l>i}}(-q)^{-|(i, l) \cap I|}[I \backslash l \sqcup i \mid J] X_{l j} .
$$

We again multiply by $q^{-1}$, and note that

$$
q^{-1}(-q)^{-|(i, l) \cap I|}=-(-q)^{-|[i, l] \cap I|} .
$$

Thus, (4-5) recovers [Goodearl and Lenagan 2002, Lemma A.2(c), Equation (A.3)] (this is the second equation of [Parshall and Wang 1991, Lemma 4.5.1(4)], in present notation).

Finally, consider the case when $i \notin I$ and $j \notin J$. We may assume that $I \sqcup i=$ $J \sqcup j=\{1, \ldots, n\}$. If we write $\hat{s}=\{1, \ldots, n\} \backslash\{s\}$ for $s=1, \ldots, n$, then (4-2) yields

$$
X_{i j}[\hat{i} \mid \hat{j}]+\hat{q} \sum_{\substack{l \in I \\ l<i}}(-q)^{l+1-i} X_{l j}[\hat{l} \mid \hat{j}]=[\hat{i} \mid \hat{j}] X_{i j}+\hat{q} \sum_{\substack{l \in J \\ l>j}}(-q)^{j+1-l}[\hat{i} \mid \hat{l}] X_{i l} .
$$

Multiplying (4-6) by $q^{-1}$ and then interchanging $q \leftrightarrow q^{-1}$ recovers the fourth equation of [Parshall and Wang 1991, Lemma 5.1.2].

Remark 4.4. As mentioned above, (4-3) could also have been obtained by setting $a=[I \mid J]$ and $b=X_{i j}$ in (2-7) and proceeding as with (4-2). In fact, interchanging any choice of $a$ and $b$ in (2-7) has the same effect as applying $\tau$, as we now explain.

First, apply $\tau$ to (2-7), and use (2-15) for both $a$ and $b$. This yields

$$
\sum_{(a),(b)} \mathbf{r}\left(a_{1}, b_{1}\right) \tau(a)_{1} \tau(b)_{1}=\sum_{(a),(b)} \mathbf{r}\left(a_{2}, b_{2}\right) \tau(b)_{2} \tau(a)_{2} .
$$

Invoking Lemma 2.7 and setting $a^{\prime}=\tau(a)$ and $b^{\prime}=\tau(b)$, (4-7) becomes

$$
\sum_{\left(a^{\prime}\right),\left(b^{\prime}\right)} \mathbf{r}\left(b_{2}^{\prime}, a_{2}^{\prime}\right) a_{1}^{\prime} b_{1}^{\prime}=\sum_{\left(a^{\prime}\right),\left(b^{\prime}\right)} \mathbf{r}\left(b_{1}^{\prime}, a_{1}^{\prime}\right) b_{2}^{\prime} a_{2}^{\prime} .
$$


Equation (4-8) is nothing but (2-7) with $a$ and $b$ replaced by $b^{\prime}$ and $a^{\prime}$, respectively.

Similarly, applying the anti-automorphism $\tau_{2}$ to (2-7) and relabelling again recovers (2-7) with $a$ and $b$ interchanged.

4.5. Two further commutation relations. Each case of commutation relations for $X_{i j}$ and $[I \mid J]$ derived in [Parshall and Wang 1991] has four subcases — two pairs in which one equation of each pair is obtained from the other by inserting a $q$ Laplace relation. Two commutation relations from each group of four correspond to our equations (4-2) and (4-3). It is more efficient to derive the remaining two by applying the isomorphism $\beta$ of Section 2.6, as follows. Set

$$
A^{\prime}=\mathrm{O}_{q^{-1}}\left(M_{n}(k)\right)
$$

and recall the notation $X_{i j}^{\prime}$ and $[I \mid J]^{\prime}$ for generators and quantum minors in $A^{\prime}$.

First, consider the relation (4-2) in $A^{\prime}$, but replace $i, j, I, J$ by $\tilde{i}, \tilde{j}, \tilde{I}, \tilde{J}$, respectively. The result is

$$
\begin{aligned}
& q^{-\delta(\tilde{i}, \tilde{I})} X_{\tilde{i} \tilde{j}}^{\prime}[\tilde{I} \mid \tilde{J}]^{\prime}+(1-\delta(\tilde{i}, \tilde{I}))(-\hat{q}) \sum_{\substack{\tilde{l} \in \tilde{I} \\
\tilde{l}<\tilde{i}}}(-q)^{|(\tilde{l}, \tilde{i}) \cap \tilde{I}|} X_{\tilde{l} \tilde{j}}^{\prime}[\tilde{I} \backslash \tilde{l} \sqcup \tilde{i} \mid \tilde{J}]^{\prime} \\
& =q^{-\delta(\tilde{j}, \tilde{J})}[\tilde{I} \mid \tilde{J}]^{\prime} X_{\tilde{i} \tilde{j}}^{\prime}+(1-\delta(\tilde{j}, \tilde{J}))(-\hat{q}) \sum_{\substack{\tilde{l} \in \tilde{J} \\
\tilde{l}>\tilde{j}}}(-q)^{|(\tilde{j}, \tilde{l}) \cap \tilde{J}|}[\tilde{I} \mid \tilde{J} \backslash \tilde{l} \sqcup \tilde{j}]^{\prime} X_{\tilde{i} \tilde{l}}^{\prime} \\
& \tilde{i}=\omega_{0}(i), \\
& \tilde{j}=\omega_{0}(j), \\
& \tilde{l}=\omega_{0}(l), \\
& \tilde{I}=\omega_{0}(I) \text {, } \\
& \tilde{J}=\omega_{0}(J),
\end{aligned}
$$

Now set

and apply $\beta^{-1}$ to (4-9). This yields

$$
\begin{aligned}
& q^{-\delta(i, I)} X_{i j}[I \mid J]+(\delta(i, I)-1) \hat{q} \sum_{\substack{l \in I \\
l>i}}(-q)^{|(i, l) \cap I|} X_{l j}[I \backslash l \sqcup i \mid J] \\
& \quad=q^{-\delta(j, J)}[I \mid J] X_{i j}+(\delta(j, J)-1) \hat{q} \sum_{\substack{l \in J \\
l<j}}(-q)^{|(l, j) \cap J|}[I \mid J \backslash l \sqcup j] X_{i l} .
\end{aligned}
$$

Similarly, the relation (4-3) in $A^{\prime}$ can be written

$$
\begin{gathered}
q^{-\delta(\tilde{j}, \tilde{J})} X_{\tilde{i} \tilde{j}}^{\prime}[\tilde{I} \mid \tilde{J}]^{\prime}+(1-\delta(\tilde{j}, \tilde{J}))(-\hat{q}) \sum_{\substack{\tilde{l} \in \tilde{\tilde{J}} \\
\tilde{l}<\tilde{j}}}(-q)^{|(\tilde{l}, \tilde{j}) \cap \tilde{J}|} X_{\tilde{i} \tilde{l}}^{\prime}[\tilde{I} \mid \tilde{J} \backslash \tilde{l} \sqcup \tilde{j}]^{\prime} \\
=q^{-\delta(\tilde{i}, \tilde{I})}[\tilde{I} \mid \tilde{J}]^{\prime} X_{\tilde{i} \tilde{j}}^{\prime}+(1-\delta(\tilde{i}, \tilde{I}))(-\hat{q}) \sum_{\substack{\tilde{l} \in \tilde{I} \\
\tilde{l}>\tilde{i}}}(-q)^{|(\tilde{i}, \tilde{l}) \cap \tilde{I}|}[\tilde{I} \backslash \tilde{l} \sqcup \tilde{i} \mid \tilde{J}]^{\prime} X_{\tilde{l} \tilde{j}}^{\prime}
\end{gathered}
$$


Applying $\beta^{-1}$ to (4-11) as above, we conclude that

$$
\begin{gathered}
q^{-\delta(j, J)} X_{i j}[I \mid J]+(\delta(j, J)-1) \hat{q} \sum_{\substack{l \in J \\
l>j}}(-q)^{|(j, l) \cap J|} X_{i l}[I \mid J \backslash l \sqcup j] \\
=q^{-\delta(i, I)}[I \mid J] X_{i j}+(\delta(i, I)-1) \hat{q} \sum_{\substack{l \in I \\
l<i}}(-q)^{|(l, i) \cap I|}[I \backslash l \sqcup i \mid J] X_{l j} .
\end{gathered}
$$

4.6. Quasicommutation. Elements $a, b \in A$ are said to quasicommute or $q$-commute provided they commute up to a power of $q$, that is, $a b=q^{m} b a$ for some integer $m$. The relations (2-6) say that two of the standard generators for $A$ which have the same row (or column) indices must quasicommute, and it is natural to expect other instances of this in A. From the results above, we can recover the quasicommutation relations for quantum minors given by Krob and Leclerc [1995]. These apply to certain quantum minors whose row (or column) index sets are disjoint. Cases allowing nondisjoint index sets were obtained by Leclerc and Zelevinsky [1998, Lemmas 2.1-2.3] by applying quantum Plücker relations. Building on the results of [Leclerc and Zelevinsky 1998], Scott [2005, Theorems 1 and 2] determined exactly which pairs of quantum minors quasicommute, and calculated the corresponding relations. We will recover some other cases of his results in Corollary 6.5.

First, consider $X_{i j}$ and $[M \mid N]$, with $i \in M$. If $j<\min (N)$, then either (4-3) or (4-10) implies that $X_{i j}[M \mid N]=q[M \mid N] X_{i j}$, while if $j>\max (N)$, then, by either (4-2) or (4-12), $X_{i j}[M \mid N]=q^{-1}[M \mid N] X_{i j}$. Of course, if $j \in N$, then $X_{i j}[M \mid N]=[M \mid N] X_{i j}$.

Now suppose that $I \subseteq M$ and that $J$ and $N$ are separated in the following sense: there is a partition $J=J^{\prime} \sqcup J^{\prime \prime}$ such that

$$
\max \left(J^{\prime}\right)<\min (N) \leq \max (N)<\min \left(J^{\prime \prime}\right) .
$$

Each of the generators $X_{i_{\sigma(l)}, j_{l}}$ occurring in (2-13) quasicommutes with $[M \mid N]$ as in the previous paragraph, whence

$$
X_{i_{\sigma(1)}, j_{1}} X_{i_{\sigma(2)}, j_{2}} \cdots X_{i_{\sigma(t)}, j_{t}}[M \mid N]=q^{\left|J^{\prime}\right|-\left|J^{\prime \prime}\right|}[M \mid N] X_{i_{\sigma(1)}, j_{1}} X_{i_{\sigma(2)}, j_{2}} \cdots X_{i_{\sigma(t)}, j_{t}}
$$

for all $\sigma \in S_{t}$. Consequently, under the present hypotheses,

$$
[I \mid J][M \mid N]=q^{\left|J^{\prime}\right|-\left|J^{\prime \prime}\right|}[M \mid N][I \mid J] .
$$

This recovers [Krob and Leclerc 1995, Lemma 3.7] (after interchanging $q$ and $q^{-1}$ ). In fact, (4-13) holds when $I \subseteq M$, and $J$ and $N$ are weakly separated in the sense of [Leclerc and Zelevinsky 1998], meaning that there is a partition $J \backslash N=J^{\prime} \sqcup J^{\prime \prime}$ such that $\max \left(J^{\prime}\right)<\min (N \backslash J) \leq \max (N \backslash J)<\min \left(J^{\prime \prime}\right)$ [Leclerc and Zelevinsky 1998, Lemma 2.1]. 
Applying $\tau$ to (4-13) and relabelling, we find that

$$
[I \mid J][M \mid N]=q^{\left|I^{\prime}\right|-\left|I^{\prime \prime}\right|}[M \mid N][I \mid J]
$$

when $J \subseteq N$ and $I=I^{\prime} \sqcup I^{\prime \prime}$ with $\max \left(I^{\prime}\right)<\min (M) \leq \max (M)<\min \left(I^{\prime \prime}\right)$.

\section{Computation of $\mathbf{r}([I \mid J],[M \mid N])$}

Throughout this section, let $I, J, M, N$ denote index sets contained in the interval $\{1, \ldots, n\}$, with $|I|=|J|$ and $|M|=|N|$. Our goal is to develop a formula for $\mathbf{r}([I \mid J],[M \mid N])$.

Lemma 5.1. If $\mathbf{r}([I \mid J],[M \mid N]) \neq 0$, then $I \cap M=J \cap N$ and $I \cup M=J \cup N$.

Proof. We induct on $|I|$, starting with the case $[I \mid J]=X_{i j}$. If $i=j$, Lemma 3.1 implies that $M=N$, and the conclusion is clear. If $i \neq j$, then $i>j$ by Lemma 3.2, whence Corollary 3.5 implies that $i \in N, j \in M$, and $M \backslash j=N \backslash i$. Consequently, $I \cap M=J \cap N=\varnothing$ and $I \cup M=J \cup N$.

Now suppose that $|I| \geq 2$. If $I=J$, then Proposition 3.6 implies that $M=N$, and we are done. Hence, we may assume that $I \neq J$. Since $|I|=|J|$, there must exist an element $j \in J \backslash I$. Set $J=J_{1} \sqcup J_{2}$ with $J_{1}=\{j\}$ and $J_{2}=J \backslash j$, and write $(2-22)$ in the form

$$
\pm q^{\bullet} \mathbf{r}([I \mid J],[M \mid N])=\sum_{i \in I} \sum_{L} \pm q^{\bullet} \mathbf{r}\left(X_{i j},[M \mid L]\right) \mathbf{r}([I \backslash i \mid J \backslash j],[L \mid N])
$$

Since $\mathbf{r}([I \mid J],[M \mid N]) \neq 0$, (5-1) implies that

$$
\mathbf{r}\left(X_{i j},[M \mid L]\right) \mathbf{r}([I \backslash i \mid J \backslash j],[L \mid N]) \neq 0
$$

for some $i \in I$ and some $L$.

Note that $i \neq j$, because $j \notin I$. Equation (5-2) and Lemma 3.2 now show that $i>j$, and then Corollary 3.5 implies that $i \in L, j \in M$, and $L \backslash i=M \backslash j$. Consequently, $i \notin M$ and $j \notin L$, while $L=(L \cap M) \sqcup i$ and $M=(L \cap M) \sqcup j$. Since the second factor of (5-2) is nonzero, our induction implies that $(I \backslash i) \cap L=$ $(J \backslash j) \cap N$ and $(I \backslash i) \cup L=(J \backslash j) \cup N$. Now,

$$
I \cup(L \cap M)=(I \backslash i) \cup i \cup(L \cap M)=(I \backslash i) \cup L=(J \backslash j) \cup N,
$$

and so $I \cup M=I \cup(L \cap M) \cup j=J \cup N$. Since $j \notin I \cup L$, we see from the equation $(I \backslash i) \cup L=(J \backslash j) \cup N$ that $j \notin N$. Consequently,

$$
I \cap M=I \cap(M \backslash j)=I \cap(L \backslash i)=(I \backslash i) \cap L=(J \backslash j) \cap N=J \cap N .
$$

This establishes the induction step.

Lemma 5.2. If $I \cap M=J \cap N$ and $I \cup M=J \cup N$, then: 
(a) $I \backslash J=N \backslash M$ and $J \backslash I=M \backslash N$;

(b) $\mathbf{r}([I \mid J],[M \mid N])=q^{|I \cap M|}(-q)^{\ell(I ; J \cap N)-\ell(J ; I \cap M)} \mathbf{r}([I \backslash M \mid J \backslash N],[M \mid N])$.

Proof. (a) This follows easily from the hypotheses.

(b) Write $J=J_{1} \sqcup J_{2}$ with $J_{1}=J \backslash N$ and $J_{2}=J \cap N=I \cap M$, and recall equation (2-22). We focus first on the term on the right-hand side of (2-22) with $I_{2}=J_{2}$ and $L=N$, in which case $I_{1}=I \backslash M$. For this term, we have

$$
\begin{aligned}
& (-q)^{\ell\left(I_{1} ; I_{2}\right)} \mathbf{r}\left(\left[I_{1} \mid J_{1}\right],[M \mid L]\right) \mathbf{r}\left(\left[I_{2} \mid J_{2}\right],[L \mid N]\right) \\
& =(-q)^{\ell(I \backslash M ; J \cap N)} q^{|I \cap M|} \mathbf{r}([I \backslash M \mid J \backslash N],[M \mid N]),
\end{aligned}
$$

in view of Proposition 3.6. We claim that all other terms on the right-hand side of (2-22) vanish.

Suppose that $\mathbf{r}\left(\left[I_{1} \mid J_{1}\right],[M \mid L]\right) \mathbf{r}\left(\left[I_{2} \mid J_{2}\right],[L \mid N]\right)$ is nonzero for some $I_{1}, I_{2}, L$. Lemma 5.1 implies that $I_{2} \cap L=J_{2} \cap N=J_{2}$, and then, because $\left|I_{2}\right|=\left|J_{2}\right|$, we must have $I_{2}=J_{2}$. Consequently, Proposition 3.6 implies that $L=N$, verifying the claim. Equations (2-22) and (5-3) thus yield

$$
\begin{aligned}
(-q)^{\ell(J \backslash N ; I \cap M)} \mathbf{r}([I \mid J],[M \mid N]) & \\
& =(-q)^{\ell(I \backslash M ; J \cap N)} q^{|I \cap M|} \mathbf{r}([I \backslash M \mid J \backslash N],[M \mid N]) .
\end{aligned}
$$

Finally, we have

$$
\begin{aligned}
& \ell(I ; J \cap N)=\ell(I \backslash M ; J \cap N)+\ell(I \cap M ; J \cap N), \\
& \ell(J ; I \cap M)=\ell(J \backslash N ; I \cap M)+\ell(J \cap N ; I \cap M),
\end{aligned}
$$

and, since $I \cap M=J \cap N$, we obtain

$$
\ell(I \backslash M ; J \cap N)-\ell(J \backslash N ; I \cap M)=\ell(I ; J \cap N)-\ell(J ; I \cap M) .
$$

Part (b) follows from (5-4) and (5-5).

Lemma 5.3. If $I \cap M=J \cap N=\varnothing$ and $I \cup M=J \cup N$, then

$$
\mathbf{r}([I \mid J],[M \mid N])=(-q)^{\ell(I \cup N ; I \backslash J)-\ell(J \cup M ; J \backslash I)} \mathbf{r}([I \backslash J \mid J \backslash I],[M \backslash N \mid N \backslash M]) .
$$

Proof. Write $J=J_{1} \sqcup J_{2}$ with $J_{1}=I \cap J$ and $J_{2}=J \backslash I$, and recall (2-22). Consider the term with $I_{1}=J_{1}$ and $L=M$, in which case $I_{2}=I \backslash J$. Since $I_{1} \cap M=\varnothing$, Proposition 3.6 implies that $\mathbf{r}\left(\left[I_{1} \mid J_{1}\right],[M \mid L]\right)=1$. Thus, for this term of (2-22), we have

$$
\begin{aligned}
(-q)^{\ell\left(I_{1} ; I_{2}\right)} \mathbf{r}\left(\left[I_{1} \mid J_{1}\right],[M \mid L]\right) & \mathbf{r}\left(\left[I_{2} \mid J_{2}\right],[L \mid N]\right) \\
& =(-q)^{\ell(I \cap J ; I \backslash J)} \mathbf{r}([I \backslash J \mid J \backslash I],[M \mid N])
\end{aligned}
$$


We next claim that all other terms on the right-hand side of (2-22) vanish. Hence, suppose that $\mathbf{r}\left(\left[I_{1} \mid J_{1}\right],[M \mid L]\right) \mathbf{r}\left(\left[I_{2} \mid J_{2}\right],[L \mid N]\right) \neq 0$ for some $I_{1}, I_{2}, L$. Lemma 5.1 implies that $I_{2} \cap L=J_{2} \cap N=\varnothing$ and $I_{2} \cup L=J_{2} \cup N=(J \backslash I) \cup N$, from which it follows that $I_{2}=N \backslash L$. Now, $I_{2} \cap J \subseteq N \cap J=\varnothing$, and so $I_{2} \subseteq I \backslash J$. Since also

$$
\left|I_{2}\right|=\left|J_{2}\right|=|J \backslash I|=|I \backslash J|,
$$

we must have $I_{2}=I \backslash J$. Consequently, $I_{1}=J_{1}$, and then Proposition 3.6 implies that $L=M$. This verifies the claim. As a result, (2-22) and (5-6) combine to yield

$$
\mathbf{r}([I \mid J],[M \mid N])=(-q)^{\ell(I \cap J ; I \backslash J)-\ell(I \cap J ; J \backslash I)} \mathbf{r}([I \backslash J \mid J \backslash I],[M \mid N]) .
$$

Note that $(I \backslash J) \cap M=(J \backslash I) \cap N=\varnothing$ and $(I \backslash J) \cup M=M \cup N=(J \backslash I) \cup N$. Hence, (5-7) also holds with $I, J, M, N$ replaced by $N, M, J \backslash I, I \backslash J$, respectively. That is,

$$
\begin{aligned}
\mathbf{r}([N \mid M],[J \backslash I \mid I \backslash J]) \\
\quad=(-q)^{\ell(N \cap M ; N \backslash M)-\ell(N \cap M ; M \backslash N)} \mathbf{r}([N \backslash M \mid M \backslash N],[J \backslash I \mid I \backslash J]) .
\end{aligned}
$$

In view of Lemma $2.7,(5-8)$ can be rewritten as

$$
\begin{aligned}
& \mathbf{r}([I \backslash J \mid J \backslash I],[M \mid N]) \\
& \quad=(-q)^{\ell(N \cap M ; N \backslash M)-\ell(N \cap M ; M \backslash N)} \mathbf{r}([I \backslash J \mid J \backslash I],[M \backslash N \mid N \backslash M]) .
\end{aligned}
$$

Combining (5-7) and (5-9), we obtain

$$
\mathbf{r}([I \mid J],[M \mid N])=(-q)^{\lambda} \mathbf{r}([I \backslash J \mid J \backslash I],[M \backslash N \mid N \backslash M]),
$$

where (recalling Lemma 5.2 (a))

$$
\begin{aligned}
& \lambda= \ell(I \cap J ; I \backslash J)-\ell(I \cap J ; J \backslash I) \\
& \quad+\ell(N \cap M ; N \backslash M)-\ell(N \cap M ; M \backslash N) \\
&=\ell((I \cap J) \sqcup(M \cap N) ; I \backslash J)-\ell((I \cap J) \sqcup(M \cap N) ; J \backslash I) .
\end{aligned}
$$

Next, observe that

$$
I \cup N=(I \backslash J) \sqcup(I \cap J) \sqcup(M \cap N), \quad J \cup M=(J \backslash I) \sqcup(I \cap J) \sqcup(M \cap N) .
$$

Because $|I \backslash J|=|J \backslash I|$, we have $\ell(I \backslash J ; I \backslash J)=\ell(J \backslash I ; J \backslash I)$, and therefore

$$
\lambda=\ell(I \cup N ; I \backslash J)-\ell(J \cup M ; J \backslash I) .
$$

Equations (5-10) and (5-12) establish the lemma. 
In view of Lemmas 5.1-5.3, it remains only to calculate $\mathbf{r}([I \mid J],[M \mid N])$ in the case when

$$
(I \cup N) \cap(J \cup M)=\varnothing \quad \text { and } \quad I \cup M=J \cup N,
$$

whence $I=N$ and $J=M$. Further, because of Corollary 3.3, we may assume that $I>J$. In these cases, certain sums of powers of $-q$ appear in $\mathbf{r}([I \mid J],[M \mid N])$, and we introduce the following notation to deal with them:

5.4. Definition of $\xi_{q}(\boldsymbol{I} ; \boldsymbol{J})$. Recall that, for $d \in \mathbb{N}$, the $(-q)$-integer $[d]_{-q}$ is given by

$$
\begin{aligned}
{[d]_{-q}=\frac{(-q)^{d}-(-q)^{-d}}{(-q)-(-q)^{-1}} } & =(-q)^{d-1}+(-q)^{d-3}+\cdots+(-q)^{-(d-1)} \\
& =(-q)^{1-d}\left(1+q^{2}+q^{4}+\cdots+q^{2 d-2}\right) .
\end{aligned}
$$

Hence, $1+q^{2}+q^{4}+\cdots+q^{2 d-2}=(-q)^{d-1}[d]_{-q}$.

For index sets $I \geq J$, we define a scalar $\xi_{q}(I ; J)$ as follows: First, set $m=|I|$ and write $I=\left\{r_{1}<\cdots<r_{m}\right\}$. Then, set $d_{l}=\left|\left[1, r_{l}\right] \cap J\right|-l+1$ for $l=1, \ldots, m$, noting that $d_{l} \geq 1$ because $J \leq I$. Finally, define

$$
\xi_{q}(I ; J)=\left[d_{1}\right]_{-q}\left[d_{2}\right]_{-q} \cdots\left[d_{m}\right]_{-q}
$$

with the convention that $\xi_{q}(\varnothing ; \varnothing)=1$. When $I \cap J=\varnothing$, as in the next lemma, each $d_{l}=\ell\left(r_{l} ; J\right)-l+1$. Note that $[d]_{-q^{-1}}=[d]_{-q}$ for all $d \in \mathbb{N}$, whence $\xi_{q^{-1}}(I ; J)=\xi_{q}(I ; J)$.

Lemma 5.5. If $I>J$ and $I \cap J=\varnothing$, then

$$
\mathbf{r}([I \mid J],[J \mid I])=\hat{q}^{|I|}(-q)^{\ell(J ; I)-\ell(I ; I)} \xi_{q}(I ; J) .
$$

Proof. Set $m=|I|=|J|$, write $I=\left\{r_{1}<\cdots<r_{m}\right\}$, and set $d_{l}=\ell\left(r_{l} ; J\right)-l+1$ for $l=1, \ldots, m$ as in Section 5.4.

We proceed by induction on $m$. If $m=1$, then $J=\{j\}$ for some $j<r_{1}$, whence $\ell(J ; I)=\ell(I ; I)=0$. Moreover, $d_{1}=1$ and so $\xi_{q}(I ; J)=1$. By $(2-12)$, $\mathbf{r}([I \mid J],[J \mid I])=\mathbf{r}\left(X_{r_{1} j}, X_{j r_{1}}\right)=\hat{q}$, which verifies (5-13) in this case.

Now suppose that $m>1$. Write $I=I_{1} \sqcup I_{2}$ with $I_{1}=\left\{r_{1}\right\}$ and $I_{2}=\left\{r_{2}, \ldots, r_{m}\right\}$. Since $\ell\left(I_{1} ; I_{2}\right)=0$, equation (2-20) implies that

$$
[I \mid J]=\sum_{j \in J}(-q)^{\ell(j ; J \backslash j)} X_{r_{1} j}\left[I_{2} \mid J \backslash j\right] .
$$

Applying (2-8), we obtain

$(5-14) \quad \mathbf{r}([I \mid J],[J \mid I])$

$$
=\sum_{j \in J} \sum_{L}(-q)^{|[1, j) \cap J|} \mathbf{r}\left(X_{r_{1} j},[J \mid L]\right) \mathbf{r}\left(\left[I_{2} \mid J \backslash j\right],[L \mid I]\right) .
$$


According to Lemma 3.2 and Corollary 3.5, a nonzero term can occur on the righthand side of (5-14) only if $r_{1}>j$ and $r_{1} \in L$, as well as $J \backslash j=L \backslash r_{1}$, in which case

$$
\mathbf{r}\left(X_{r_{1} j},[J \mid L]\right)=\hat{q}(-q)^{|[1, j) \cap J|-\left|\left[1, r_{1}\right) \cap L\right|} .
$$

Now, $\left|\left[1, r_{1}\right) \cap L\right|=\left|\left[1, r_{1}\right) \cap\left(L \backslash r_{1}\right)\right|=\left|\left[1, r_{1}\right) \cap(J \backslash j)\right|=d_{1}-1$, and so

$$
\mathbf{r}\left(X_{r_{1} j},[J \mid L]\right)=\hat{q}(-q)^{1+|[1, j) \cap J|-d_{1}} .
$$

Next, note that $L=J \backslash j \sqcup r_{1}$, whence $L \cap I=\left\{r_{1}\right\}$. Consequently, $I_{2} \cap L=$ $(J \backslash j) \cap I=\varnothing$ and $I_{2} \cup L=I \cup L=(J \backslash j) \cup I$. Lemma 5.3 now implies that

$$
\mathbf{r}\left(\left[I_{2} \mid J \backslash j\right],[L \mid I]\right)=(-q)^{\lambda} \mathbf{r}\left(\left[I_{2} \mid J \backslash j\right],\left[J \backslash j \mid I_{2}\right]\right)
$$

where

$$
\begin{aligned}
\lambda & =\ell\left(I ; I_{2}\right)-\ell(L ; J \backslash j) \\
& =\ell\left(I_{2} ; I_{2}\right)-\ell(J \backslash j ; J \backslash j)+\ell\left(r_{1} ; I_{2}\right)-\ell\left(r_{1} ; J \backslash j\right)=-d_{1}+1 .
\end{aligned}
$$

Combining equations (5-14)-(5-17), we obtain

$$
\mathbf{r}([I \mid J],[J \mid I])=\hat{q} \sum_{\substack{j \in J \\ j<r_{1}}}(-q)^{2+2|[1, j) \cap J|-2 d_{1}} \mathbf{r}\left(\left[I_{2} \mid J \backslash j\right],\left[J \backslash j \mid I_{2}\right]\right) .
$$

It remains to compute $\mathbf{r}\left(\left[I_{2} \mid J \backslash j\right],\left[J \backslash j \mid I_{2}\right]\right)$ for $j \in J$ with $j<r_{1}$. Observe that $I_{2}>J \backslash j$ for any such $j$, so that our induction hypothesis will apply. Now,

$$
\begin{aligned}
\ell\left(J \backslash j ; I_{2}\right) & =\ell\left(J ; I_{2}\right)=\ell(J ; I)-\ell\left(J ; r_{1}\right)=\ell(J ; I)-m+d_{1}, \\
\ell\left(I_{2} ; I_{2}\right) & =\ell\left(I ; I_{2}\right)=\ell(I ; I)-m+1,
\end{aligned}
$$

whence $\ell\left(J \backslash j ; I_{2}\right)-\ell\left(I_{2} ; I_{2}\right)=\ell(J ; I)-\ell(I ; I)+d_{1}-1$. For $l=1, \ldots, m-1$, observe that

$$
\ell\left(r_{l+1} ; J \backslash j\right)-l+1=\ell\left(r_{l+1} ; J\right)-l=d_{l+1},
$$

and consequently $\xi_{q}\left(I_{2} ; J \backslash j\right)=\left[d_{2}\right]_{-q}\left[d_{3}\right]_{-q} \cdots\left[d_{m}\right]_{-q}$. Thus, our induction hypothesis implies that

$$
\begin{aligned}
& \mathbf{r}\left(\left[I_{2} \mid J \backslash j\right],\left[J \backslash j \mid I_{2}\right]\right) \\
& \quad=\hat{q}^{m-1}(-q)^{\ell(J ; I)-\ell(I ; I)+d_{1}-1}\left[d_{2}\right]_{-q}\left[d_{3}\right]_{-q} \cdots\left[d_{m}\right]_{-q} .
\end{aligned}
$$

Inserting (5-19) in (5-18), we obtain

$(5-20) \quad \mathbf{r}([I \mid J],[J \mid I])$

$$
=\hat{q}^{m}(-q)^{\ell(J ; I)-\ell(I ; I)+1-d_{1}}\left[d_{2}\right]_{-q}\left[d_{3}\right]_{-q} \cdots\left[d_{m}\right]_{-q} \sum_{\substack{j \in J \\ j<r_{1}}} q^{2|[1, j) \cap J|} .
$$


The summation appearing in (5-20) is just $\sum_{t=1}^{d_{1}} q^{2(t-1)}=(-q)^{d_{1}-1}\left[d_{1}\right]_{-q}$, whence

$$
\left[d_{2}\right]_{-q}\left[d_{3}\right]_{-q} \cdots\left[d_{m}\right]_{-q} \sum_{\substack{j \in J \\ j<r_{1}}} q^{2|[1, j) \cap J|}=(-q)^{d_{1}-1} \xi_{q}(I ; J) .
$$

Equations (5-20) and (5-21) establish (5-13), completing the induction step.

Theorem 5.6. Let $I, J, M, N \subseteq\{1, \ldots, n\}$ with $|I|=|J|$ and $|M|=|N|$.

(a) If $\mathbf{r}([I \mid J],[M \mid N]) \neq 0$, then

$$
I \geq J, \quad I \cap M=J \cap N, \quad I \cup M=J \cup N .
$$

(b) If conditions (5-22) hold, then

$$
\mathbf{r}([I \mid J],[M \mid N])=q^{|I \cap M|} \hat{q}^{|I \backslash J|}(-q)^{\lambda} \xi_{q}(I \backslash J ; J \backslash I),
$$

where $\lambda=\ell((J \backslash N) \cup(M \backslash I) ; I \backslash J)-\ell((J \backslash N) \cup(M \backslash I) ; J \backslash I)$.

Proof. (a) Follows from Corollary 3.3 and Lemma 5.1.

(b) Recall from Lemma 5.2 that $I \backslash J=N \backslash M$ and $J \backslash I=M \backslash N$. If $I=J$, then we must have $M=N$. In this case, $\mathbf{r}([I \mid J],[M \mid N])=q^{|I \cap M|}$ by Proposition 3.6, and we are done. Now assume that $I \neq J$, and note that $I \backslash J>J \backslash I$. We shall need the observations that

$$
\begin{aligned}
& (I \backslash M) \cup N=I \cup N, \\
& (I \backslash M) \backslash(J \backslash N)=I \backslash J, \\
& (J \backslash N) \cup M=J \cup M, \\
& (J \backslash N) \backslash(I \backslash M)=J \backslash I .
\end{aligned}
$$

Applying successively Lemmas 5.2, 5.3 and 5.5, we obtain

$$
\mathbf{r}([I \mid J],[M \mid N])=q^{|I \cap M|} \hat{q}^{|I \backslash J|}(-q)^{\lambda} \xi_{q}(I \backslash J ; J \backslash I),
$$

where

$$
\begin{aligned}
\lambda=\ell(I ; J \cap N)-\ell(J ; I \cap M) & +\ell(I \cup N ; I \backslash J) \\
& -\ell(J \cup M ; J \backslash I)+\ell(J \backslash I ; I \backslash J)-\ell(I \backslash J ; I \backslash J) .
\end{aligned}
$$

Observe that $(I \cup N) \sqcup(J \backslash I)=J \cup N=I \cup M=(J \cup M) \sqcup(I \backslash J)$, whence

$$
\begin{array}{r}
\ell(I \cup N ; I \backslash J)-\ell(J \cup M ; J \backslash I)+\ell(J \backslash I ; I \backslash J)-\ell(I \backslash J ; I \backslash J) \\
=\ell(J \cup M ; I \backslash J)-\ell(J \cup M ; J \backslash I) .
\end{array}
$$

Next, observe that $I \backslash N=J \backslash M$ and $N \backslash I=M \backslash J$. Moreover,

$$
\begin{aligned}
& I \cup M=I \cup M \cup N=I \sqcup(N \backslash I) \sqcup(M \backslash N), \\
& J \cup N=J \cup M \cup N=J \sqcup(M \backslash J) \sqcup(N \backslash M),
\end{aligned}
$$


and consequently

$$
\begin{aligned}
& \ell(I ; J \cap N)+\ell(N \backslash I ; J \cap N)+\ell(M \backslash N ; J \cap N)=\ell(I \cup M ; J \cap N), \\
& \ell(J ; I \cap M)+\ell(M \backslash J ; I \cap M)+\ell(N \backslash M ; I \cap M)=\ell(J \cup N ; I \cap M) .
\end{aligned}
$$

It follows that

$$
\begin{aligned}
\ell(I ; J \cap N)- & \ell(J ; I \cap M) \\
= & \ell(N \backslash M ; I \cap M)-\ell(M \backslash N ; J \cap N) \\
= & |N \backslash M| \cdot|I \cap M|-\ell(I \cap M ; N \backslash M) \\
& \quad-|M \backslash N| \cdot|J \cap N|+\ell(J \cap N ; M \backslash N) \\
= & \ell(I \cap M ; J \backslash I)-\ell(I \cap M ; I \backslash J) .
\end{aligned}
$$

Finally, since

$$
(J \cup M) \backslash(I \cap M)=(J \backslash(J \cap N)) \cup(M \backslash(I \cap M))=(J \backslash N) \cup(M \backslash I),
$$

we conclude from (5-25) and (5-26) that

$$
\lambda=\ell((J \backslash N) \cup(M \backslash I) ; I \backslash J)-\ell((J \backslash N) \cup(M \backslash I) ; J \backslash I) .
$$

In view of (5-24) and (5-27), the theorem is proved.

Example 5.7. Let $[I \mid J]=[45678 \mid 12345]$ and $[M \mid N]=[123459 \mid 456789]$, where we have omitted commas between elements of the index sets. It is clear that $I \geq J$; moreover, $I \cap M=\{4,5\}=J \cap N$ and $I \cup M=\{1, \ldots, 9\}=J \cup N$. Hence, conditions (5-22) hold. Now $I \backslash J=\{6,7,8\}$ and $J \backslash I=\{1,2,3\}$, while $(J \backslash N) \cup(M \backslash I)=$ $\{1,2,3,9\}$, whence

$$
\ell((J \backslash N) \cup(M \backslash I) ; I \backslash J)-\ell((J \backslash N) \cup(M \backslash I) ; J \backslash I)=3-6=-3 .
$$

Since all the elements of $I \backslash J$ are greater than all the elements of $J \backslash I$, we have

$$
\xi_{q}(I \backslash J ; J \backslash I)=[3]_{-q}[2]_{-q}[1]_{-q}=\left(q^{2}+1+q^{-2}\right)\left(-q-q^{-1}\right) .
$$

Thus, we conclude from (5-23) that

$$
\mathbf{r}([I \mid J],[M \mid N])=q^{2} \hat{q}^{3}(-q)^{-3}\left(q^{2}+1+q^{-2}\right)\left(-q-q^{-1}\right) .
$$

\section{General commutation relations}

Now that we have formulas for the value of the braiding form $\mathbf{r}$ on pairs of quantum minors, commutation relations follow readily from property (2-7). The following notation for certain index sets and exponents will be helpful in displaying the results. Recall the quantities $\ell(\cdot, \cdot)$ and $\xi_{q}(\cdot, \cdot)$ from Section 2.8 and Section 5.4. 
6.1. Definitions of index sets $\{<X \| Y\}$ and $\{>X \| Y\}$ and numerical quantities $\mathscr{L}(\boldsymbol{S}, \boldsymbol{X}, \boldsymbol{Y})$ and $\mathscr{L}^{\natural}(\boldsymbol{T}, \boldsymbol{X}, \boldsymbol{Y})$. For any subsets $X$ and $Y$ of $\{1, \ldots, n\}$, define

$$
\begin{aligned}
& \{<X \| Y\}=\{S \subseteq X \cup Y|X \cap Y \subseteq S ;| S|=| X \mid ; S<X\}, \\
& \{>X \| Y\}=\{T \subseteq X \cup Y|X \cap Y \subseteq T ;| T|=| X \mid ; T>X\} .
\end{aligned}
$$

In Section 7, we shall need index sets $\{\leq X \| Y\}$ and $\{\geq X \| Y\}$, defined in a similar manner. For any set $S \subseteq X \cup Y$ such that $X \cap Y \subseteq S$, define

$$
S^{\natural}=S_{X, Y}^{\natural}=(X \cap Y) \sqcup((X \cup Y) \backslash S) .
$$

Note that, if $S \in\{<X \| Y\}$ or $S \in\{>X \| Y\}$, then $\left|S^{\natural}\right|=|Y|$. Finally, for $S \in\{<X \| Y\}$ and $T \in\{>X \| Y\}$, define

$$
\begin{aligned}
& \mathscr{L}(S, X, Y)=\ell\left(\left(S \backslash S^{\natural}\right) \cup(Y \backslash X) ; X \backslash S\right)-\ell\left(\left(S \backslash S^{\natural}\right) \cup(Y \backslash X) ; S \backslash X\right), \\
& \mathscr{L}^{\natural}(T, X, Y) \\
& \quad=\ell\left(\left(T^{\natural} \backslash T\right) \cup(X \backslash Y) ; T \backslash X\right)-\ell\left(\left(T^{\natural} \backslash T\right) \cup(X \backslash Y) ; X \backslash T\right) .
\end{aligned}
$$

For example, suppose that $X=\{2,3,4,6\}$ and $Y=\{1,3,5\}$. Then $\{<X \| Y\}$ consists of those 4-element subsets $S$ of $\{1, \ldots, 6\}$ such that $3 \in S$ and $S<X$. There are six such sets:

$$
\begin{array}{lll}
\{1,2,3,4\}, & \{1,2,3,5\}, & \{1,2,3,6\}, \\
\{1,3,4,5\}, & \{1,3,4,6\}, & \{2,3,4,5\} .
\end{array}
$$

Similarly, $\{>X \| Y\}$ consists of those 4-element subsets $T$ of $\{1, \ldots, 6\}$ such that $3 \in T$ and $T>X$. There are two: $\{3,4,5,6\}$ and $\{2,3,5,6\}$. Finally, consider the set $S=\{1,2,3,4\} \in\{<X \| Y\}$. Then $S^{\natural}=\{3,5,6\}$, and so

$$
\mathscr{L}(S, X, Y)=\ell(\{1,2,4,5\} ;\{6\})-\ell((\{1,2,4,5\} ;\{1\})=0-3 .
$$

Theorem 6.2. If $I, J, M, N \subseteq\{1, \ldots, n\}$ with $|I|=|J|$ and $|M|=|N|$, then

where

$$
\begin{gathered}
q^{|I \cap M|}[I \mid J][M \mid N]+q^{|I \cap M|} \sum_{S \in\{<I \| M\}} \lambda_{S}[S \mid J]\left[S^{\natural} \mid N\right] \\
=q^{|J \cap N|}[M \mid N][I \mid J]+q^{|J \cap N|} \sum_{T \in\{>J \| N\}} \mu_{T}\left[M \mid T^{\natural}\right][I \mid T],
\end{gathered}
$$

$$
\begin{aligned}
\lambda_{S} & =\hat{q}^{|I \backslash S|}(-q)^{\mathscr{L}(S, I, M)} \xi_{q}(I \backslash S ; S \backslash I) \\
\mu_{T} & =\hat{q}^{|T \backslash J|}(-q)^{\mathscr{L}^{\natural}(T, J, N)} \xi_{q}(T \backslash J ; J \backslash T)
\end{aligned}
$$

for $S \in\{<I \| M\}$ and $T \in\{>J \| N\}$. 
Proof. Taking $a=[I \mid J]$ and $b=[M \mid N]$ in (2-7), we obtain

$$
\begin{gathered}
\sum_{\substack{|S|=|I| \\
\left|S^{\prime}\right|=|M|}} \mathbf{r}\left([I \mid S],\left[M \mid S^{\prime}\right]\right)[S \mid J]\left[S^{\prime} \mid N\right] \\
=\sum_{\substack{|T|=|J| \\
\left|T^{\prime}\right|=|N|}} \mathbf{r}\left([T \mid J],\left[T^{\prime} \mid N\right]\right)\left[M \mid T^{\prime}\right][I \mid T] .
\end{gathered}
$$

In view of Corollary 3.3 and Lemma 5.1, the left-hand summation in (6-6) can be restricted to index sets $S$ and $S^{\prime}$ such that

$$
\begin{aligned}
|S| & =|I|, & I & \geq S, \\
I \cap M & =S \cap S^{\prime}, & I \cup M & =S \cup S^{\prime} .
\end{aligned}
$$

Proposition 3.6 shows that the coefficient of the term with $S=I$ and $S^{\prime}=M$ is $q^{|I \cap M|}$, and that the terms with $S=I$ and $S^{\prime} \neq M$ vanish.

The index sets $S$ and $S^{\prime}$ such that $S \neq I$ and (6-7) hold are precisely those for which $S \in\{<I \| M\}$ and $S^{\prime}=S^{\natural}$. For these index sets, Theorem 5.6 shows that

$$
\mathbf{r}\left([I \mid S],\left[M \mid S^{\prime}\right]\right)=q^{|I \cap M|} \lambda_{S} .
$$

Thus, the left-hand side of (6-6) reduces to the left-hand side of (6-4).

Similarly, the right-hand side of (6-6) reduces to the right-hand side of (6-4), and the theorem is proved.

Corollary 6.3. If $I, J, M, N \subseteq\{1, \ldots, n\}$ with $|I|=|J|$ and $|M|=|N|$, then

where

$$
\begin{aligned}
& q^{|J \cap N|}[I \mid J][M \mid N]+q^{|J \cap N|} \sum_{S \in\{<J \| N\}} \lambda_{S}[I \mid S]\left[M \mid S^{\natural}\right] \\
& =q^{|I \cap M|}[M \mid N][I \mid J]+q^{|I \cap M|} \sum_{T \in\{>I \| M\}} \mu_{T}\left[T^{\natural} \mid N\right][T \mid J],
\end{aligned}
$$

$$
\begin{aligned}
\lambda_{S} & =\hat{q}^{|J \backslash S|}(-q)^{\mathscr{L}(S, J, N)} \xi_{q}(J \backslash S ; S \backslash J) \\
\mu_{T} & =\hat{q}^{|T \backslash I|}(-q)^{\mathscr{L}^{\natural}(T, I, M)} \xi_{q}(T \backslash I ; I \backslash T)
\end{aligned}
$$

for $S \in\{<J \| N\}$ and $T \in\{>I \| M\}$.

Proof. Interchange the index sets in the statement of Theorem 6.2 as follows: $I \leftrightarrow J$ and $M \leftrightarrow N$. Then apply the automorphism $\tau$ to the resulting version of (6-4) to obtain (6-8) (recall (2-16)).

This corollary can also be obtained from Theorem 6.2 by interchanging $I \leftrightarrow M$ and $J \leftrightarrow N$, in which case one should also interchange $S \leftrightarrow T^{\natural}$ and $T \leftrightarrow S^{\natural}$. 
6.4. Further quasicommutation. In particular, Theorem 6.2 yields quasicommutation relations of the form $q^{|I \cap M|}[I \mid J][M \mid N]=q^{|J \cap N|}[M \mid N][I \mid J]$ in cases where the index sets $\{<I \| M\}$ and $\{>J \| N\}$ are empty. This occurs, for instance, if either

$$
[I \mid J]=[1, \ldots, r \mid n+1-r, \ldots, n] \quad \text { or } \quad[M \mid N]=[n+1-r, \ldots, n \mid 1, \ldots, r],
$$

recovering the well-known fact that the northeastern-most and southwestern-most quantum minors are normal elements of $A$. Moreover,

(6-10) $[1, \ldots, r \mid J][M \mid 1, \ldots, s]$

$$
=q^{|J \cap[1, s]|-|[1, r] \cap M|}[M \mid 1, \ldots, s][1, \ldots, r \mid J],
$$

which is part of [Hodges and Levasseur 1994, Proposition 1.1] (with $q^{2}$ replaced by $q$ ). Also, (6-10) immediately implies the type A case of [Berenstein and Zelevinsky 2005, Equation 10.3].

We record the general quasicommutation relations of the above type in the next corollary. Part (a) recovers one case of [Scott 2005, Theorem 2]. It does not seem, however, that the relations (4-13) and (4-14) follow directly from equations such as (6-4) or (6-8).

Corollary 6.5. Let $I, J, M, N \subseteq\{1, \ldots, n\}$ with $|I|=|J|$ and $|M|=|N|$.

(a) If $\max (M \backslash I)<\min (I \backslash M)$ and $\max (J \backslash N)<\min (N \backslash J)$, then

$$
[I \mid J][M \mid N]=q^{|I \cap M|-|J \cap N|}[M \mid N][I \mid J] .
$$

(b) If $\max (I \backslash M)<\min (M \backslash I)$ and $\max (N \backslash J)<\min (J \backslash N)$, then

$$
[I \mid J][M \mid N]=q^{|J \cap N|-|I \cap M|}[M \mid N][I \mid J] .
$$

Proof. (a) If $S \in\{<J \| N\}$, then $S \backslash(J \cap N)<J \backslash N$, whence

$$
\max (S \backslash(J \cap N)) \leq \max (J \backslash N)<\min (N \backslash J) .
$$

But then $S$ is disjoint from $N \backslash J$. Since $J \cap N \subseteq S \subseteq J \cup N$ and $|S|=|J|$, this forces $S=J$, which is ruled out by the assumption that $S<J$. Thus, $\{<J \| N\}=\varnothing$. Similarly, $\{>I \| M\}=\varnothing$, and thus (6-11) follows from (6-8).

(b) Interchange $I \leftrightarrow M$ and $J \leftrightarrow N$, and apply part (a).

Example 6.6 $(n=6)$. Let $J=N=\{1,2,3\}$, and take $I=\{1,4,5\}$ and $M=$ $\{2,3,6\}$. We first apply Theorem 6.2. Note that $\{>J \| N\}$ is empty because $J=N$. For $S \in\{<I \| M\}$, we make the calculations in Table 1, where commas have been deleted for the sake of abbreviation (for instance, $\{123\}$ stands for the index set $\{1,2,3\})$. 


\begin{tabular}{l|ccccc}
\hline$S$ & $\{123\}$ & $\{124\}$ & $\{125\}$ & $\{134\}$ & $\{135\}$ \\
$S^{\natural}$ & $\{456\}$ & $\{356\}$ & $\{346\}$ & $\{256\}$ & $\{246\}$ \\
$I \backslash S$ & $\{45\}$ & $\{5\}$ & $\{4\}$ & $\{5\}$ & $\{4\}$ \\
$S \backslash I$ & $\{23\}$ & $\{2\}$ & $\{2\}$ & $\{3\}$ & $\{3\}$ \\
$\left(S \backslash S^{\natural}\right) \cup(M \backslash I)$ & $\{1236\}$ & $\{12346\}$ & $\{12356\}$ & $\{12346\}$ & $\{12356\}$ \\
$\ell\left(\left(S \backslash S^{\natural}\right) \cup(M \backslash I) ; I \backslash S\right)$ & 2 & 1 & 2 & 1 & 2 \\
$\ell\left(\left(S \backslash S^{\natural}\right) \cup(M \backslash I) ; S \backslash I\right)$ & 3 & 3 & 3 & 2 & 2 \\
$\mathscr{L}(S, I, M)$ & -1 & -2 & -1 & -1 & 0 \\
$\xi_{q}(I \backslash S ; S \backslash I)$ & $-q-q^{-1}$ & 1 & 1 & 1 & 1 \\
\hline
\end{tabular}

Table 1

Consequently, Theorem 6.2 implies that

(6-13) $q^{3}[236 \mid J][145 \mid J]$

$$
\begin{aligned}
&=[145 \mid J][236 \mid J]+\hat{q}^{2}(-q)^{-1}\left(-q-q^{-1}\right)[123 \mid J][456 \mid J] \\
&+ \hat{q}(-q)^{-2}[124 \mid J][356 \mid J]+\hat{q}(-q)^{-1}[125 \mid J][346 \mid J] \\
&+\hat{q}(-q)^{-1}[134 \mid J][256 \mid J]+\hat{q}[135 \mid J][246 \mid J] .
\end{aligned}
$$

The relation (6-13) matches the one calculated by Fioresi [1999, Example 2.22] (see the first display on page 435, where one must replace $q$ by $q^{-1}$ to account for the difference between (2-6) and the relations used in that paper).

For contrast, we record the relation obtained from Corollary 6.3 for the current choices of $I, J, M, N$ :

$(6-14) \quad q^{3}[145 \mid J][236 \mid J]$

$$
\begin{aligned}
&=[236 \mid J][145 \mid J]+\hat{q}[235 \mid J][146 \mid J] \\
&+\hat{q}(-q)^{-1}[234 \mid J][156 \mid J]+\hat{q}[136 \mid J][245 \mid J] \\
&+\hat{q}^{2}[135 \mid J][246 \mid J]+\hat{q}^{2}(-q)^{-1}[134 \mid J][256 \mid J] \\
&+\hat{q}(-q)^{-1}[126 \mid J][345 \mid J]+\hat{q}^{2}(-q)^{-1}[125 \mid J][346 \mid J] \\
&+\hat{q}^{2}(-q)^{-2}[124 \mid J][356 \mid J]+\hat{q}(-q)^{-4}[123 \mid J][456 \mid J] .
\end{aligned}
$$

We derive two further relations from Theorem 6.2 and Corollary 6.3 with the help of the isomorphism $\beta$ of Section 2.6, as in Section 4.5. For use in the upcoming proof, note that, since $\omega_{0}$ reverses inequalities of integers, it also reverses the 
ordering on index sets: if $U$ and $V$ are subsets of $\{1, \ldots, n\}$ with $|U|=|V|$, then $U \leq V$ if and only if $\omega_{0} U \geq \omega_{0} V$.

Theorem 6.7. If $I, J, M, N \subseteq\{1, \ldots, n\}$ with $|I|=|J|$ and $|M|=|N|$, then

where

$$
\begin{aligned}
& q^{|J \cap N|}[I \mid J][M \mid N]+q^{|J \cap N|} \sum_{S \in\{>I \| M\}} \tilde{\mu}_{S}[S \mid J]\left[S^{\natural} \mid N\right] \\
& =q^{|I \cap M|}[M \mid N][I \mid J]+q^{|I \cap M|} \sum_{T \in\{<J \| N\}} \tilde{\lambda}_{T}\left[M \mid T^{\natural}\right][I \mid T],
\end{aligned}
$$

$$
\begin{aligned}
& \tilde{\mu}_{S}=(-\hat{q})^{|S \backslash I|}(-q)^{-\mathscr{L}^{\natural}(S, I, M)} \xi_{q}(S \backslash I ; I \backslash S) \\
& \tilde{\lambda}_{T}=(-\hat{q})^{|J \backslash T|}(-q)^{-\mathscr{L}(T, J, N)} \xi_{q}(J \backslash T ; T \backslash J)
\end{aligned}
$$

for $S \in\{>I \| M\}$ and $T \in\{<J \| N\}$.

Proof. Just for this proof, write $\tilde{U}=\omega_{0} U$ for index sets $U$, and observe that

$$
\omega_{0}(\{>I \| M\})=\{<\tilde{I} \| \tilde{M}\} \quad \text { and } \quad \omega_{0}(\{<J \| N\})=\{>\tilde{J} \| \tilde{N}\} .
$$

Note also that $\tilde{S}^{\natural}=\widetilde{S^{\natural}}$ for $S \in\{>I \| M\}$, and similarly $\tilde{T}^{\natural}=\widetilde{T^{\natural}}$ for $T \in\{<J \| N\}$.

Set $A^{\prime}=O_{q^{-1}}\left(M_{n}(k)\right)$, with generators $X_{i j}^{\prime}$ and braiding form $\mathbf{r}^{\prime}$, and label the quantum minors in $A^{\prime}$ by $[I \mid J]^{\prime}$. Recall the isomorphism $\beta: A \rightarrow A^{\prime}$ from Section 2.6, and equation (2-17). Note that, when specializing general results to $A^{\prime}$, the scalars $q$ and $\hat{q}$ change to $q^{-1}$ and $-\hat{q}$, respectively.

Now apply Theorem 6.2 to the quantum minors $[\tilde{I} \mid \tilde{J}]^{\prime}$ and $[\tilde{M} \mid \tilde{N}]^{\prime}$ in $A^{\prime}$ :

where

$$
\begin{aligned}
& q^{-|\tilde{I} \cap \tilde{M}|}[\tilde{I} \mid \tilde{J}]^{\prime}[\tilde{M} \mid \tilde{N}]^{\prime}+q^{-|\tilde{I} \cap \tilde{M}|} \sum_{S \in\{>I \| M\}} \lambda_{\tilde{S}}^{\prime}[\tilde{S} \mid \tilde{J}]^{\prime}\left[\tilde{S} \tilde{S}^{\natural} \mid \tilde{N}\right]^{\prime} \\
& \quad=q^{-|\tilde{J} \cap \tilde{N}|}[\tilde{M} \mid \tilde{N}]^{\prime}[\tilde{I} \mid \tilde{J}]^{\prime}+q^{-|\tilde{J} \cap \tilde{N}|} \sum_{T \in\{<J \| N\}} \mu_{\tilde{T}}^{\prime}\left[\tilde{M} \mid \tilde{T}^{\natural}\right]^{\prime}[\tilde{I} \mid \tilde{T}]^{\prime}
\end{aligned}
$$

$$
\begin{aligned}
\lambda_{\tilde{S}}^{\prime} & =(-\hat{q})^{|I \backslash S|}(-q)^{-\mathscr{L}(\tilde{S}, \tilde{I}, \tilde{M})} \xi_{q}(\tilde{I} \backslash \tilde{S} ; \tilde{S} \backslash \tilde{I}), \\
\mu_{\tilde{T}}^{\prime} & =(-\hat{q})^{|T \backslash J|}(-q)^{-\mathscr{L}^{\natural}(\tilde{T}, \tilde{J}, \tilde{N})} \xi_{q}(\tilde{T} \backslash \tilde{J} ; \tilde{J} \backslash \tilde{T}),
\end{aligned}
$$

for $S \in\{>I \| M\}$ and $T \in\{<J \| N\}$. (Here we have simplified the exponents of the $-\hat{q}$ terms and used the observation that $\xi_{q^{-1}}(U ; V)=\xi_{q}(U ; V)$ for any $\left.U, V.\right)$ Applying the isomorphism $\beta^{-1}$ to (6-17) yields, in $A$,

$$
\begin{aligned}
& q^{-|I \cap M|}[I \mid J][M \mid N]+q^{-|I \cap M|} \sum_{S \in\{>I \| M\}} \lambda_{\tilde{S}}^{\prime}[S \mid J]\left[S^{\natural} \mid N\right] \\
& =q^{-|J \cap N|}[M \mid N][I \mid J]+q^{-|J \cap N|} \sum_{T \in\{<J \| N\}} \mu_{\tilde{T}}^{\prime}\left[M \mid T^{\natural}\right][I \mid T] .
\end{aligned}
$$


Equation (6-15) will follow from (6-18) once we see that $\lambda_{\tilde{S}}^{\prime}=\tilde{\mu}_{S}$ and $\mu_{\tilde{T}}^{\prime}=\tilde{\lambda}_{T}$ for all $S$ and $T$.

Take $S \in\{>I \| M\}$ and observe that

$$
\begin{aligned}
S \cap S^{\natural} & =I \cap M, & & S \cup S^{\natural}=I \cup M, \\
S^{\natural} \backslash M & =I \backslash S, & & M \backslash S^{\natural}=S \backslash I .
\end{aligned}
$$

It follows from Theorem 5.6 and Lemma 2.7 that

$$
q^{-|I \cap M|} \lambda_{\tilde{S}}^{\prime}=q^{-|\tilde{I} \cap \tilde{M}|} \lambda_{\tilde{S}}^{\prime}=\mathbf{r}^{\prime}\left([\tilde{I} \mid \tilde{S}]^{\prime},\left[\tilde{M} \mid \tilde{S}^{\natural}\right]^{\prime}\right)=\mathbf{r}^{\prime}\left(\left[M \mid S^{\natural}\right]^{\prime},[I \mid S]^{\prime}\right) .
$$

With the help of (6-19), a second application of Theorem 5.6 shows that

$$
\mathbf{r}^{\prime}\left(\left[M \mid S^{\natural}\right]^{\prime},[I \mid S]^{\prime}\right)=q^{-|I \cap M|} \tilde{\mu}_{S},
$$

and therefore $\lambda_{\tilde{S}}^{\prime}=\tilde{\mu}_{S}$. Similarly, $\mu_{\tilde{T}}^{\prime}=\tilde{\lambda}_{T}$ for all $T \in\{<J \| N\}$, and the theorem is proved.

The next corollary is obtained from Theorem 6.7 in the same way as was Corollary 6.3 from Theorem 6.2.

Corollary 6.8. If $I, J, M, N \subseteq\{1, \ldots, n\}$ with $|I|=|J|$ and $|M|=|N|$, then

$$
\begin{aligned}
& q^{|I \cap M|}[I \mid J][M \mid N]+q^{|I \cap M|} \sum_{S \in\{>J \| N\}} \tilde{\mu}_{S}[I \mid S]\left[M \mid S^{\natural}\right] \\
& =q^{|J \cap N|}[M \mid N][I \mid J]+q^{|J \cap N|} \sum_{T \in\{<I \| M\}} \tilde{\lambda}_{T}\left[T^{\natural} \mid N\right][T \mid J],
\end{aligned}
$$

where

$$
\begin{aligned}
& \tilde{\mu}_{S}=(-\hat{q})^{|S \backslash J|}(-q)^{-\mathscr{L}^{\natural}(S, J, N)} \xi_{q}(S \backslash J ; J \backslash S) \\
& \tilde{\lambda}_{T}=(-\hat{q})^{|I \backslash T|}(-q)^{-\mathscr{L}(T, I, M)} \xi_{q}(I \backslash T ; T \backslash I)
\end{aligned}
$$

for $S \in\{>J \| N\}$ and $T \in\{<I \| M\}$.

\section{Some variants}

Consider the general form of a commutation relation for quantum minors $[I \mid J]$ and $[M \mid N]$, namely, an equation that allows a product $[I \mid J][M \mid N]$ to be replaced by a scalar multiple of the reverse product $[M \mid N][I \mid J]$, at the cost of some additional terms. In an equation such as (6-4), the additional terms are of two types: scalar multiples of $[S \mid J]\left[S^{\natural} \mid N\right]$ and of $\left[M \mid T^{\natural}\right][I \mid T]$. In some applications, one type may be more useful than the other. For instance, the prefered bases constructed in [Goodearl and Lenagan 2000] consist of certain products of quantum minors in which quantum minors with larger index sets must occur to the left of those with smaller index sets. Thus, if $|I|<|M|$, then $[M \mid N][I \mid J]$ and the terms $\left[M \mid T^{\natural}\right][I \mid T]$ are in preferred order, but $[I \mid J][M \mid N]$ and the terms $[S \mid J]\left[S^{\natural} \mid N\right]$ 
are not. A commutation relation in which all the extra terms are in preferred order can be achieved by iteration: after a first application of (6-4), apply (6-4) to any products $[S \mid J]\left[S^{\natural} \mid N\right]$ that appear, and continue until all terms have the desired form. This produces a relation in which $q^{|I \cap M|}[I \mid J][M \mid N]$ is expressed as $q^{|J \cap N|}[M \mid N][I \mid J]$ plus a linear combination of products $\left[S^{\natural} \mid T^{\natural}\right][S \mid T]$, where $S \in\{\leq I \| M\}$ and $T \in\{\geq J \| N\}$. We begin by illustrating the iteration process in the next example.

The aim of this section is to derive closed formulas (that is, without iterations) for commutation relations of the type just discussed.

Example 7.1 $(n=4)$. Consider $[I \mid J]=[23 \mid 12]$ and $[M \mid N]=[14 \mid 23]$. First, (6-4) leads to the relation

$$
\begin{aligned}
& {[23 \mid 12][14 \mid 23]-q[14 \mid 23][23 \mid 12]} \\
& \quad=q \hat{q}[14 \mid 12][23 \mid 23]-\hat{q}(-q)^{-1}[12 \mid 12][34 \mid 23]-\hat{q}[13 \mid 12][24 \mid 23]
\end{aligned}
$$

The last two terms on the right-hand side of (7-1) must now be treated. Applying (6-4) in each case, we obtain

$$
\begin{aligned}
& {[12 \mid 12][34 \mid 23]=q[34 \mid 23][12 \mid 12]+q \hat{q}[34 \mid 12][12 \mid 23]} \\
& {[13 \mid 12][24 \mid 23]=q[24 \mid 23][13 \mid 12]+q \hat{q}[24 \mid 12][13 \mid 23]}
\end{aligned}
$$

Note that (7-3) contains a term involving [12|12] [34|23]. Hence, we first substitute that equation into (7-1), and then combine the two [12|12] [34|23]-terms, before substituting (7-2) into the result. The final relation is

$$
\begin{aligned}
& {[23 \mid 12][14 \mid 23]-q[14 \mid 23][23 \mid 12]} \\
& \begin{aligned}
=q \hat{q}[14 \mid 12][23 \mid 23] & -\hat{q} q[24 \mid 23][13 \mid 12]-\hat{q}^{2} q[24 \mid 12][13 \mid 23] \\
& +\hat{q} q^{2}[34 \mid 23][12 \mid 12]+\hat{q}^{2} q^{2}[34 \mid 12][12 \mid 23]
\end{aligned}
\end{aligned}
$$

In each of the terms on the right-hand side of (7-4), the second factor is of the form $[S \mid T]$, where $S \in\{23,13,12\}=\{\leq I \| M\}$ and $T \in\{23,12\}=\{\geq J \| N\}$.

Lemma 7.2. Let $s \in\{1, \ldots, n-1\}$, and let $B$ and $C$ be the subalgebras of $A=$ $\mathrm{O}_{q}\left(M_{n}(k)\right)$ given by

$$
B=k\left\langle X_{i j} \mid 1 \leq i \leq n, 1 \leq j \leq s\right\rangle \quad \text { and } \quad C=k\left\langle X_{i j} \mid 1 \leq i \leq n, s+1 \leq j \leq n\right\rangle .
$$

The multiplication map $\mu: B \otimes_{k} C \rightarrow A$ is a vector space isomorphism. 
Proof. Let $X, Y$, and $Z$ be the standard PBW bases of the respective algebras $B$, $C$, and $A$. Thus,

$$
\begin{aligned}
& X=\left\{\left(X_{11}^{b_{11}} \cdots X_{1 s}^{b_{1 s}}\right)\left(X_{21}^{b_{21}} \cdots X_{2 s}^{b_{2 s}}\right) \cdots\left(X_{n 1}^{b_{n 1}} \cdots X_{n s}^{b_{n s}}\right) \mid b_{i j} \in \mathbb{Z}^{+}\right\}, \\
& Y=\left\{\left(X_{1, s+1}^{c_{1, s+1}} \cdots X_{1 n}^{c_{1 n}}\right)\left(X_{2, s+1}^{c_{2, s+1}} \cdots X_{2 n}^{c_{2 n}}\right) \cdots\left(X_{n, s+1}^{c_{n, s+1}} \cdots X_{n n}^{c_{n n}}\right) \mid c_{i j} \in \mathbb{Z}^{+}\right\}, \\
& Z=\left\{\left(X_{11}^{a_{11}} \cdots X_{1 n}^{a_{1 n}}\right)\left(X_{21}^{a_{21}} \cdots X_{2 n}^{a_{2 n}}\right) \cdots\left(X_{n 1}^{a_{n 1}} \cdots X_{n n}^{a_{n n}}\right) \mid a_{i j} \in \mathbb{Z}^{+}\right\},
\end{aligned}
$$

where the variables occur in each monomial in lexicographic order. Observe that the monomials $X_{i 1}^{b_{i 1}} \cdots X_{i s}^{b_{i s}}$ and $X_{l, s+1}^{c_{l, s+1}} \cdots X_{l n}^{c_{l n}}$ commute whenever $i>l$. Hence, any product of a monomial from $X$ with a monomial from $Y$ can be rewritten as

$$
\begin{gathered}
\left(\left(X_{11}^{b_{11}} \cdots X_{1 s}^{b_{1 s}}\right)\left(X_{21}^{b_{21}} \cdots X_{2 s}^{b_{2 s}}\right) \cdots\left(X_{n 1}^{b_{n 1}} \cdots X_{n s}^{b_{n s}}\right)\right) \\
\quad \times\left(\left(X_{1, s+1}^{c_{1, s+1}} \cdots X_{1 n}^{c_{1 n}}\right) \cdot\left(X_{2, s+1}^{c_{2, s+1}} \cdots X_{2 n}^{c_{2 n}}\right) \cdots\left(X_{n, s+1}^{c_{n, s+1}} \cdots X_{n n}^{c_{n n}}\right)\right) \\
=\left(X_{11}^{b_{11}} \cdots X_{1 s}^{b_{1 s}}\right)\left(X_{1, s+1}^{c_{1, s+1}} \cdots X_{1 n}^{c_{1 n}}\right)\left(X_{21}^{b_{21}} \cdots X_{2 s}^{b_{2 s}}\right) \\
\quad \times\left(X_{2, s+1}^{c_{2, s+1}} \cdots X_{2 n}^{c_{2 n}}\right) \cdots\left(X_{n 1}^{b_{n 1}} \cdots X_{n s}^{b_{n s}}\right)\left(X_{n, s+1}^{c_{n, s+1}} \cdots X_{n n}^{c_{n n}}\right) .
\end{gathered}
$$

Consequently, $\mu$ maps the set $\{x \otimes y \mid x \in X, y \in Y\}$ bijectively onto $Z$, and the lemma follows.

Theorem 7.3. If $I, J, M, N \subseteq\{1, \ldots, n\}$ with $|I|=|J|$ and $|M|=|N|$, then

$$
\begin{aligned}
& q^{|I \cap M|}[I \mid J][M \mid N] \\
& =q^{|J \cap N|}[M \mid N][I \mid J]+q^{|J \cap N|} \sum_{\substack{S \in\{\leq I \| M\} \\
T \in\{\geq J \| N\} \\
(S, T) \neq(I, J)}} \tilde{\lambda}_{S} \mu_{T}\left[S^{\natural} \mid T^{\natural}\right][S \mid T],
\end{aligned}
$$

where

$$
\begin{aligned}
\tilde{\lambda}_{S} & =(-\hat{q})^{|I \backslash S|}(-q)^{-\mathscr{L}(S, I, M)} \xi_{q}(I \backslash S ; S \backslash I) \\
\mu_{T} & =\hat{q}^{|T \backslash J|}(-q)^{\mathscr{L}^{\natural}(T, J, N)} \xi_{q}(T \backslash J ; J \backslash T)
\end{aligned}
$$

for $S \in\{\leq I \| M\}$ and $T \in\{\geq J \| N\}$.

Remark 7.4. We have isolated the term $q^{|J \cap N|}[M \mid N][I \mid J]$ on the right-hand side of (7-5) to emphasize that this equation is a commutation relation. It may, of course, be incorporated in the given summation as a term where $(S, T)=(I, J)$, since $\tilde{\lambda}_{I} \mu_{J}=1$.

Proof of Theorem 7.3. Note that the coefficients $\lambda_{S}$ and $\mu_{T}$ defined in (6-5) also depend on $I, J, M, N$. For purposes of the present proof, we record that dependence by writing

$$
\begin{aligned}
& \lambda_{S}^{X, Y}=\hat{q}^{|X \backslash S|}(-q)^{\mathscr{L}(S, X, Y)} \xi_{q}(X \backslash S ; S \backslash X), \\
& \mu_{T}^{J, N}=\hat{q}^{|T \backslash J|}(-q)^{\mathscr{L}^{\natural}(T, J, N)} \xi_{q}(T \backslash J ; J \backslash T),
\end{aligned}
$$


for $S \in\{\leq X \| Y\}$ and $T \in\{\geq J \| N\}$. Note that $\lambda_{X}^{X, Y}=1$ and $\mu_{J}^{J, N}=1$. For $S \in\{<I \| M\}$, set

$$
\alpha_{S}^{I, M}=\sum_{\substack{S_{1} \in\{<I \| M\} \\ S_{2} \in\left\{<S_{1} \| S_{1}^{\natural}\right\} \\ S \in\left\{<S_{i-1} \| S_{i-1}^{\natural}\right\}}}(-1)^{i} \lambda_{S_{1}}^{I, M} \lambda_{S_{2}}^{S_{1}, S_{1}^{\natural}} \cdots \lambda_{S}^{S_{i-1}, S_{i-1}^{\natural}},
$$

where, in terms with $i=1$, we interpret $S_{0}=I$ and $S_{0}^{\natural}=M$. Finally, set $\alpha_{I}^{I, M}=1$. We claim that

$$
q^{|I \cap M|}[I \mid J][M \mid N]=q^{|J \cap N|} \sum_{\substack{S \in\{\leq I \| M\} \\ T \in\{\geq J \| N\}}} \alpha_{S}^{I, M} \mu_{T}^{J, N}\left[S^{\natural} \mid T^{\natural}\right][S \mid T] .
$$

Let $t=|I|$, and let $\mathcal{N}_{t}$ denote the collection of $t$-element subsets of $\{1, \ldots, n\}$, partially ordered as in Section 2.10. For proving (7-7), we proceed by induction on $I$ relative to the ordering in $\mathcal{N}_{t}$. To start, suppose that $I$ is minimal in $\mathcal{N}_{t}$ (that is, $I=\{1, \ldots, t\})$. In this case, $\{<I \| M\}$ is empty, and so Theorem 6.2 implies that

$$
q^{|I \cap M|}[I \mid J][M \mid N]=q^{|J \cap N|}[M \mid N][I \mid J]+q^{|J \cap N|} \sum_{T \in\{>J \| N\}} \mu_{T}^{J, N}\left[M \mid T^{\natural}\right][I \mid T],
$$

which verifies (7-7).

Now suppose that $I$ is not minimal in $\mathcal{N}_{t}$, but that (7-7) holds whenever $I$ is replaced by an index set $I^{\prime}<I$. Theorem 6.2 implies that

$$
\begin{aligned}
& q^{|I \cap M|}[I \mid J][M \mid N] \\
& \quad=q^{|J \cap N|} \sum_{T \in\{\geq J \| N\}} \mu_{T}^{J, N}\left[M \mid T^{\natural}\right][I \mid T]-q^{|I \cap M|} \sum_{S_{1} \in\{<I \| M\}} \lambda_{S_{1}}^{I, M}\left[S_{1} \mid J\right]\left[S_{1}^{\natural} \mid N\right] .
\end{aligned}
$$

Recall that $S_{1} \cap S_{1}^{\natural}=I \cap M$ for $S_{1} \in\{<I \| M\}$, by definition of $S_{1}^{\natural}$. Hence, our induction hypothesis yields

$$
q^{|I \cap M|}\left[S_{1} \mid J\right]\left[S_{1}^{\natural} \mid N\right]=q^{|J \cap N|} \sum_{\substack{S \in\left\{\leq S_{1} \| S_{1}^{\natural}\right\} \\ T \in\{\geq J \| N\}}} \alpha_{S}^{S_{1}, S_{1}^{\natural}} \mu_{T}^{J, N}\left[S^{\natural} \mid T^{\natural}\right][S \mid T]
$$

for all $S_{1} \in\{<I \| M\}$. Substitute (7-9) in (7-8), which yields

$$
\begin{gathered}
q^{|I \cap M|}[I \mid J][M \mid N]=q^{|J \cap N|} \sum_{T \in\{\geq J \| N\}} \mu_{T}^{J, N}\left[M \mid T^{\natural}\right][I \mid T] \\
-q^{|J \cap N|} \sum_{\substack{S_{1} \in\{<I \| M\} \\
S \in\left\{\leq S_{1} \| S_{1}^{\natural}\right\} \\
T \in\{\geq J \| N\}}} \lambda_{S_{1}}^{I, M} \alpha_{S}^{S_{1}, S_{1}^{\natural}} \mu_{T}^{J, N}\left[S^{\natural} \mid T^{\natural}\right][S \mid T] .
\end{gathered}
$$


Since $\alpha_{I}^{I, M}=1$, the coefficients in the first summation of (7-10) match the corresponding coefficients in (7-7). The second summation of (7-10) may be rewritten in the form

where each

$$
q^{|J \cap N|} \sum_{\substack{S \in\{<I \| M\} \\ T \in\{\geq J \| N\}}} \beta_{S} \mu_{T}^{J, N}\left[S^{\natural} \mid T^{\natural}\right][S \mid T],
$$

$$
\beta_{S}=-\sum_{\substack{S_{1} \in\{<I \| M\} \\ S \in\left\{\leq S_{1} \| S_{1}^{\natural}\right\}}} \lambda_{S_{1}}^{I, M} \alpha_{S}^{S_{1}, S_{1}^{\natural}}=\alpha_{S}^{I, M} .
$$

Thus, (7-10) yields (7-7), establishing the induction step. This proves (7-7).

It remains to show that $\alpha_{S}^{I, M}=\tilde{\lambda}_{S}$ for $S \in\{\leq I \| M\}$.

Observe that all quantities appearing in (7-7) involve index sets contained in the union $I \cup J \cup M \cup N$, and so they remain the same if we work in $O_{q}\left(M_{v}(k)\right)$ for some $v>n$. Hence, there is no loss of generality in assuming that $n \geq|I|+|M|$. If we set

$$
J^{*}=\{n-|I|+1, \ldots, n\} \quad \text { and } \quad N^{*}=\{1, \ldots,|M|\},
$$

we have $\max \left(N^{*}\right)<\min \left(J^{*}\right)$. Note also that $J^{*}$ is maximal among $|I|$-element subsets of $\{1, \ldots, n\}$. The quantum minors $\left[U \mid N^{*}\right]$, for $U \subseteq\{1, \ldots, n\}$ with $|U|=$ $|M|$, are homogeneous elements of distinct degrees with respect to the grading on $A$ discussed in Section 2.2. Hence, the $\left[U \mid N^{*}\right]$ are linearly independent over $k$. Similarly, the $\left[V \mid J^{*}\right]$, for $V \subseteq\{1, \ldots, n\}$ with $|V|=|I|$, are linearly independent, and thus it follows from Lemma 7.2 that the products $\left[U \mid N^{*}\right]\left[V \mid J^{*}\right]$ are linearly independent over $k$.

Now apply (7-7) to the quantum minors $\left[I \mid J^{*}\right]$ and $\left[M \mid N^{*}\right]$. Since $\left\{>J^{*} \| N^{*}\right\}$ is empty, we obtain

$$
q^{|I \cap M|}\left[I \mid J^{*}\right]\left[M \mid N^{*}\right]=\sum_{S \in\{\leq I \| M\}} \alpha_{S}^{I, M}\left[S^{\natural} \mid N^{*}\right]\left[S \mid J^{*}\right] .
$$

However, we also have a relation of this type from Corollary 6.8, which may be written in the form

$$
q^{|I \cap M|}\left[I \mid J^{*}\right]\left[M \mid N^{*}\right]=\sum_{T \in\{\leq I \| M\}} \tilde{\lambda}_{T}\left[T^{\natural} \mid N^{*}\right]\left[T \mid J^{*}\right] .
$$

Since the products $\left[S^{\natural} \mid N^{*}\right]\left[S \mid J^{*}\right]$ are linearly independent, it follows from (7-11) and (7-12) that $\alpha_{S}^{I, M}=\tilde{\lambda}_{S}$ for all $S \in\{\leq I \| M\}$. Therefore (7-7) implies (7-5), as desired.

As is easily checked, Theorem 7.3 directly yields equation (7-4).

We next consider the derivation of new relations from Theorem 7.3. Unlike the situation in Section 5, however, the methods used there to prove Corollary 6.3 and 
Theorem 6.7 yield the same result when applied to Theorem 7.3. Hence, we use the method of Corollary 6.3.

Corollary 7.5. If $I, J, M, N \subseteq\{1, \ldots, n\}$ with $|I|=|J|$ and $|M|=|N|$, then

$$
\begin{aligned}
& q^{|J \cap N|}[I \mid J][M \mid N] \\
& =q^{|I \cap M|}[M \mid N][I \mid J]+q^{|I \cap M|} \sum_{\substack{S \in\{\geq I \| M\} \\
T \in\{\leq J \| N\} \\
(S, T) \neq(I, J)}} \mu_{S} \tilde{\lambda}_{T}\left[S^{\natural} \mid T^{\natural}\right][S \mid T],
\end{aligned}
$$

where

$$
\begin{aligned}
& \mu_{S}=\hat{q}^{|S \backslash I|}(-q)^{\mathscr{L}^{\natural}(S, I, M)} \xi_{q}(S \backslash I ; I \backslash S) \\
& \tilde{\lambda}_{T}=(-\hat{q})^{|J \backslash T|}(-q)^{-\mathscr{L}(T, J, N)} \xi_{q}(J \backslash T ; T \backslash J)
\end{aligned}
$$

for $S \in\{\geq I \| M\}$ and $T \in\{\leq J \| N\}$.

Proof. Interchange $I \leftrightarrow J$ and $M \leftrightarrow N$ in the statement of Theorem 7.3, and also interchange the roles of $S$ and $T$ in the summation. This yields

$$
\begin{aligned}
& q^{|J \cap N|}[J \mid I][N \mid M] \\
& =q^{|I \cap M|}[N \mid M][J \mid I]+q^{|I \cap M|} \sum_{\substack{T \in\{\leq J \| N\} \\
S \in\{\leq I \| M\} \\
(T, S) \neq(J, I)}} \tilde{\lambda}_{T}^{J, N} \mu_{S}^{I, M}\left[T^{\natural} \mid S^{\natural}\right][T \mid S],
\end{aligned}
$$

where we have placed the superscripts on $\tilde{\lambda}_{T}^{J, N}$ and $\mu_{S}^{I, M}$ as reminders of the changes required when carrying over (7-6) to the present situation. Thus, observe that $\tilde{\lambda}_{T}^{J, N}$ and $\mu_{S}^{I, M}$ are equal to the scalars denoted $\tilde{\lambda}_{T}$ and $\mu_{S}$ in (7-14). Consequently, an application of the automorphism $\tau$ to (7-15) yields (7-13) (recall (2-16)).

Remark 7.6. In addition to (7-5) and (7-13), one can derive two commutation relations for quantum minors $[I \mid J]$ and $[M \mid N]$ in which the additional terms involve products in the same order as $[I \mid J][M \mid N]$, rather than in reverse order. To obtain such results, simply interchange the roles of $[I \mid J]$ and $[M \mid N]$ in Theorem 7.3 and Corollary 7.5. One may wish to simplify the coefficients; for instance, with the help of observations such as (6-19), one sees that

$$
\mathscr{L}\left(S^{\natural}, M, I\right)=\mathscr{L}^{\natural}(S, I, M) .
$$

We leave this to the interested reader.

Example $7.7(n=4)$. We close the section by applying Corollary 7.5 to the quantum minors $[I \mid J]=[23 \mid 13]$ and $[M \mid N]=[14 \mid 24]$. In this case, equation (7-13) 
becomes

$[23 \mid 13][14 \mid 24]$

$$
\begin{aligned}
=[14 \mid 24][23 \mid 13] & +\hat{q}[13 \mid 24][24 \mid 13]+\hat{q}(-q)^{-1}[12 \mid 24][34 \mid 13] \\
+ & (-\hat{q})[14 \mid 34][23 \mid 12]+\hat{q}(-\hat{q})[13 \mid 34][24 \mid 12] \\
& +\hat{q}(-q)^{-1}(-\hat{q})[12 \mid 34][34 \mid 12] .
\end{aligned}
$$

Equation (7-16) matches the relation calculated by Fioresi [2004, Example 6.2] (after replacing $q$ by $q^{-1}$ ).

\section{Poisson brackets}

In this final section, we use the commutation relations for quantum minors obtained above to derive expressions for the standard Poisson bracket on pairs of classical minors in $O\left(M_{n}(k)\right)$. In particular, we recover, for the case of the standard bracket, a formula calculated by Kupershmidt [1994]. Although the study of Poisson brackets is often restricted to characteristic zero, that restriction is not needed for the results below.

8.1. Standard Poisson bracket on $O\left(M_{n}(k)\right)$. Recall that a Poisson bracket on a commutative $k$-algebra $B$ is a $k$-bilinear map $\{\cdot, \cdot\}: B \times B \rightarrow B$ such that

- $B$ is a Lie algebra with respect to $\{\cdot, \cdot\}$; and

- $\{b, \cdot\}$ is a derivation for each $b \in B$.

Note that a Poisson bracket is uniquely determined by its values on pairs of elements from a $k$-algebra generating set for $B$.

Write $\mathcal{O}\left(M_{n}(k)\right)$ as a commutative polynomial ring over $k$ in indeterminates $x_{i j}$ for $i, j=1, \ldots, n$. The standard Poisson bracket on this algebra is the unique Poisson bracket such that

$$
\begin{array}{ll}
\left\{x_{i j}, x_{l j}\right\}=x_{i j} x_{l j} & \text { if } i<l, \\
\left\{x_{i j}, x_{i m}\right\}=x_{i j} x_{i m} & \text { if } j<m, \\
\left\{x_{i j}, x_{l m}\right\}=0 & \text { if } i<l, j>m, \\
\left\{x_{i j}, x_{l m}\right\}=2 x_{i m} x_{l j} & \text { if } i<l, j<m .
\end{array}
$$

8.2. $O_{q}\left(M_{n}\right)$ as a quantization of $O\left(M_{n}\right)$. It is well known that $O_{q}\left(M_{n}(K)\right)$, for a rational function field $K=k(q)$, is a quantization of the Poisson algebra $O\left(M_{n}(k)\right)$, in the sense that the Poisson bracket on $\mathcal{O}\left(M_{n}(k)\right)$ is the "semiclassical limit" (as $q \rightarrow 1$ ) of the scaled commutator bracket $\frac{1}{q-1}[\cdot, \cdot]$ on $O_{q}\left(M_{n}(K)\right)$; we indicate the details below. 
For the remainder of this section, replace the scalar $q$ by an indeterminate, and consider the quantum matrix algebra

$$
\mathrm{O}_{q}\left(M_{n}(k(q))\right),
$$

defined over the rational function field $k(q)$.

The $k\left[q^{ \pm 1}\right]$-subalgebra $A_{0}$ of $O_{q}\left(M_{n}(k(q))\right)$ generated by the $X_{i j}$ can be presented, as a $k\left[q^{ \pm 1}\right]$-algebra, by the generators $X_{i j}$ and relations (2-6), from which it follows that there is an isomorphism

$$
A_{0} /(q-1) A_{0} \stackrel{\cong}{\rightrightarrows} \mathcal{O}\left(M_{n}(k)\right)
$$

sending the cosets

$$
X_{i j}+(q-1) A_{0} \longmapsto x_{i j} \quad \text { for all } i, j .
$$

We identify $A_{0} /(q-1) A_{0}$ with $\mathcal{O}\left(M_{n}(k)\right)$ via (8-2). Since $\mathcal{O}\left(M_{n}(k)\right)$ is commutative, the additive commutator $[\cdot, \cdot]$ on $A_{0}$ takes all its values in $(q-1) A_{0}$, and so $\frac{1}{q-1}[\cdot, \cdot]$ is well-defined on $A_{0}$. It follows that the latter bracket induces a well-defined Poisson bracket on $\mathcal{O}\left(M_{n}(k)\right)$, such that

$$
\{\bar{a}, \bar{b}\}=\overline{(a b-b a) /(q-1)}
$$

for $a, b \in A_{0}$, where overbars denote cosets modulo $(q-1) A_{0}$. This induced bracket is nothing but the standard Poisson bracket on $\mathcal{O}\left(M_{n}(k)\right)$, as one easily sees by computing its values on pairs of generators $x_{i j}, x_{l m}$.

We shall apply (8-3) when $\bar{a}$ and $\bar{b}$ are minors. In order to reserve the notation $[I \mid J]$ for classical minors, we denote the quantum minors in $O_{q}\left(M_{n}(k(q))\right)$ by

$$
[I \mid J]_{q} .
$$

Note that $[I \mid J]_{q}$ is an element of $A_{0}$, and that the isomorphism (8-2) maps the coset of $[I \mid J]_{q}$ to $[I \mid J]$. Hence, for pairs of minors, (8-3) can be written as

$$
\{[I \mid J],[M \mid N]\}=\overline{\left([I \mid J]_{q}[M \mid N]_{q}-[M \mid N]_{q}[I \mid J]_{q}\right) /(q-1)} .
$$

Combining (8-4) with formulas for additive commutators of quantum minors thus yields formulas for Poisson brackets of classical minors. For instance, from (6-10) we obtain

$$
\begin{aligned}
& \{[1, \ldots, r \mid J],[M \mid 1, \ldots, s]\}= \\
& \quad(|[1, r] \cap J|-|M \cap[1, s]|)[1, \ldots, r \mid J][M \mid 1, \ldots, s],
\end{aligned}
$$

which recovers some cases of [Kogan and Zelevinsky 2002, Theorem 2.6]. 
Theorem 8.3. If $I, J, M, N \subseteq\{1, \ldots, n\}$ with $|I|=|J|$ and $|M|=|N|$, then

$(8-6) \quad\{[I \mid J],[M \mid N]\}$

$$
\begin{aligned}
=(|J \cap N|-|I \cap M|)[I \mid J][M \mid N] & \\
& +2 \sum_{\substack{j \in J \backslash N \\
n \in N \backslash J \\
j<n}}(-1)^{|(J \Delta N) \cap(j, n)|}[I \mid J \sqcup n \backslash j][M \mid N \sqcup j \backslash n] \\
& -2 \sum_{\substack { i \in I \backslash M \\
\begin{subarray}{c}{i \in M \backslash I \\
i>m{ i \in I \backslash M \\
\begin{subarray} { c } { i \in M \backslash I \\
i > m } }\end{subarray}}(-1)^{|(I \Delta M) \cap(m, i)|}[I \sqcup m \backslash i \mid J][M \sqcup i \backslash m \mid N] .
\end{aligned}
$$

Proof. Write (6-4) in the form

$$
\begin{aligned}
& {[I \mid J]_{q}[M \mid N]_{q}-[M \mid N]_{q}[I \mid J]_{q}} \\
& =\left(q^{|J \cap N|-|I \cap M|}-1\right)[M \mid N]_{q}[I \mid J]_{q} \\
& \quad+q^{|J \cap N|-|I \cap M|} \sum_{T \in\{>J \| N\}} \mu_{T}\left[M \mid T^{\natural}\right]_{q}[I \mid T]_{q}-\sum_{S \in\{<I \| M\}} \lambda_{S}[S \mid J]_{q}\left[S^{\natural} \mid N\right]_{q} .
\end{aligned}
$$

Since $\hat{q}^{2} /(q-1)$ vanishes modulo $q-1$, we only need to consider the terms in the sums for $T \in\{>J \| N\}$ with $|T \backslash J|=1$, and $S \in\{<I \| M\}$ with $|I \backslash S|=1$. Any such $T$ has the form

$$
T=J \sqcup n \backslash j
$$

with $j \in J \backslash N$ and $n \in N \backslash J$ such that $j<n$, whence

$$
T^{\natural}=N \sqcup j \backslash n \quad \text { and } \quad\left(T^{\natural} \backslash T\right) \cup(J \backslash N)=(J \Delta N) \backslash n,
$$

and so

$$
\begin{aligned}
\mathscr{L}^{\natural}(T, J, N) & =\ell((J \Delta N) \backslash n ; n)-\ell((J \Delta N) \backslash n ; j) \\
& =\ell(J \Delta N ; n)-\ell(J \Delta N ; j)+1=-|(J \Delta N) \cap(j, n)| .
\end{aligned}
$$

Similarly, the indices $S$ that appear have the form

$$
S=I \sqcup m \backslash i
$$

with $i \in I \backslash M$ and $m \in M \backslash I$ such that $i>m$, whence

$$
S^{\natural}=M \sqcup i \backslash m \quad \text { and } \quad \mathscr{L}(S, I, M)=-|(I \Delta M) \cap(m, i)| .
$$

Consequently, dividing (8-7) by $q-1$, and then reducing the resulting equation modulo $q-1$ yields (8-6).

Similarly, Corollary 6.3 yields: 
Theorem 8.4. If $I, J, M, N \subseteq\{1, \ldots, n\}$ with $|I|=|J|$ and $|M|=|N|$, then

$$
\begin{aligned}
& \{[I \mid J],[M \mid N]\} \\
& =(|I \cap M|-|J \cap N|)[I \mid J][M \mid N] \\
& +2 \sum_{\substack{i \in I \backslash M \backslash M \\
m \in M \backslash I \\
i<m}}(-1)^{|(I \Delta M) \cap(i, m)|}[I \sqcup m \backslash i \mid J][M \sqcup i \backslash m \mid N] \\
& -2 \sum_{\substack{j \in J \backslash N \\
n \in N \backslash J \\
j>n}}(-1)^{|(J \Delta N) \cap(n, j)|}[I \mid J \sqcup n \backslash j][M \mid N \sqcup j \backslash n] .
\end{aligned}
$$

Finally, provided $k$ does not have characteristic 2, we can average equations $(8-6)$ and (8-8) to obtain:

Corollary 8.5. Let $I, J, M, N \subseteq\{1, \ldots, n\}$ with $|I|=|J|$ and $|M|=|N|$. If char $k \neq 2$, then

$$
\begin{aligned}
\{[I \mid J],[M \mid N]\}= & \sum_{\substack{i \in I \backslash M \\
m \in M \backslash I \backslash \\
i<m}}(-1)^{|(I \Delta M) \cap(i, m)|}[I \sqcup m \backslash i \mid J][M \sqcup i \backslash m \mid N] \\
& -\sum_{\substack{i \in I \backslash M \\
m \in M \backslash I \\
i>m}}(-1)^{|(I \Delta M) \cap(m, i)|}[I \sqcup m \backslash i \mid J][M \sqcup i \backslash m \mid N] \\
& +\sum_{\substack{j \in J \backslash N \\
n \in N \backslash J \\
j<n}}(-1)^{|(J \Delta N) \cap(j, n)|}[I \mid J \sqcup n \backslash j][M \mid N \sqcup j \backslash n] \\
& -\sum_{\substack{j \in J \backslash N \\
n \in N \backslash J \\
j>n}}(-1)^{|(J \Delta N) \cap(n, j)|}[I \mid J \sqcup n \backslash j][M \mid N \sqcup j \backslash n] .
\end{aligned}
$$

Equation (8-9) is the standard case of the formula of Kupershmidt [1994, Equation (9)]. To obtain the standard Poisson bracket in his setting, make the following choices for the structure constants:

$$
r_{l m}^{i j}=\left\{\begin{aligned}
1 & \text { if } i>j, l=j, m=i, \\
-1 & \text { if } i<j, l=j, m=i, \\
0 & \text { otherwise }
\end{aligned}\right.
$$




\section{Acknowledgments}

We thank T. H. Lenagan, L. Rigal, A. Zelevinsky, and the referee for their comments and suggestions concerning this project.

\section{References}

[Berenstein and Zelevinsky 2005] A. Berenstein and A. Zelevinsky, "Quantum cluster algebras", Adv. Math. 195:2 (2005), 405-455. MR 2006a:20092 Zbl 02189412

[Brown and Goodearl 2002] K. A. Brown and K. R. Goodearl, Lectures on algebraic quantum groups, Advanced Courses in Mathematics. CRM Barcelona, Birkhäuser Verlag, Basel, 2002. MR 2003f:16067 Zbl 1027.17010

[Fioresi 1999] R. Fioresi, "Quantum deformation of the Grassmannian manifold", J. Algebra 214:2 (1999), 418-447. MR 2000j:20092 Zbl 0955.17007

[Fioresi 2004] R. Fioresi, "Commutation relations among quantum minors in $O_{q}\left(M_{n}(k)\right)$ ", J. Algebra 280:2 (2004), 655-682. MR 2005f:20086 Zbl 1072.16035

[Goodearl and Lenagan 2000] K. R. Goodearl and T. H. Lenagan, "Quantum determinantal ideals", Duke Math. J. 103:1 (2000), 165-190. MR 2001k:16080 Zbl 0958.16025

[Goodearl and Lenagan 2002] K. R. Goodearl and T. H. Lenagan, "Prime ideals invariant under winding automorphisms in quantum matrices", International J. Math. 13:5 (2002), 497-532. MR 2003h:20091 Zbl 1054.16030

[Goodearl and Lenagan 2003] K. R. Goodearl and T. H. Lenagan, "Winding-invariant prime ideals in quantum $3 \times 3$ matrices”, J. Algebra 260:2 (2003), 657-687. MR 2004g:20069 Zbl 1059.16035

[Hayashi 1992] T. Hayashi, "Quantum groups and quantum determinants", J. Algebra 152:1 (1992), 146-165. MR 93j:17031 Zbl 0906.17011

[Hodges and Levasseur 1993] T. J. Hodges and T. Levasseur, "Primitive ideals of $\mathbf{C}_{q}$ [SL(3)]", Comm. Math. Phys. 156:3 (1993), 581-605. MR 94k:17023 Zbl 0801.17012

[Hodges and Levasseur 1994] T. J. Hodges and T. Levasseur, "Primitive ideals of $\mathbf{C}_{q}$ [SL $(n)$ ]", $J$. Algebra 168:2 (1994), 455-468. MR 95i:16038 Zbl 0814.17012

[Hodges et al. 1997] T. J. Hodges, T. Levasseur, and M. Toro, "Algebraic structure of multiparameter quantum groups”, Adv. Math. 126:1 (1997), 52-92. MR 98e:17022 Zbl 0878.17009

[Joseph 1995] A. Joseph, Quantum groups and their primitive ideals, vol. 29, Ergebnisse der Mathematik und ihrer Grenzgebiete (3) [Results in Mathematics and Related Areas (3)], Springer, Berlin, 1995. MR 96d:17015 Zbl 0808.17004

[Kassel 1995] C. Kassel, Quantum groups, Graduate Texts in Mathematics 155, Springer, New York, 1995. MR 96e:17041 Zbl 0808.17003

[Klimyk and Schmüdgen 1997] A. Klimyk and K. Schmüdgen, Quantum groups and their representations, Texts and Monographs in Physics, Springer, Berlin, 1997. MR 99f:17017 Zbl 0891.17010

[Kogan and Zelevinsky 2002] M. Kogan and A. Zelevinsky, "On symplectic leaves and integrable systems in standard complex semisimple Poisson-Lie groups”, Int. Math. Res. Not. 32 (2002), 1685-1702. MR 2003k:53107 Zbl 1006.22015

[Krob and Leclerc 1995] D. Krob and B. Leclerc, "Minor identities for quasi-determinants and quantum determinants", Comm. Math. Phys. 169:1 (1995), 1-23. MR 96g:15015 Zbl 0829.15024

[Kupershmidt 1994] B. A. Kupershmidt, "Poisson relations between minors and their consequences”, J. Phys. A 27:13 (1994), L507-L513. MR 95g:22033 Zbl 0842.17018 
[Lakshmibai and Reshetikhin 1991] V. Lakshmibai and N. Reshetikhin, "Quantum deformations of flag and Schubert schemes", Comptes Rendus de l'Académie des Sciences. Sér. I Math. 313:3 (1991), 121-126. MR 92f:14053 Zbl 0753.17021

[Lakshmibai and Reshetikhin 1992] V. Lakshmibai and N. Reshetikhin, "Quantum flag and Schubert schemes", pp. 145-181 in Deformation theory and quantum groups with applications to mathematical physics (Amherst, MA, 1990), edited by M. Gerstenhaber and J. Stasheff, Contemp. Math. 134, Amer. Math. Soc., Providence, RI, 1992. MR 94a:14055 Zbl 0792.17012

[Lambe and Radford 1997] L. A. Lambe and D. E. Radford, Introduction to the quantum YangBaxter equation and quantum groups: an algebraic approach, Mathematics and its Applications 423, Kluwer Academic Publishers, Dordrecht, 1997. MR 1483071 (98k:16054) Zbl 0888.16021

[Larson and Towber 1991] R. G. Larson and J. Towber, "Two dual classes of bialgebras related to the concepts of 'quantum group' and 'quantum Lie algebra'”, Comm. Algebra 19:12 (1991), 3295-3345. MR 93b:16070 Zbl 0751.16014

[Leclerc and Zelevinsky 1998] B. Leclerc and A. Zelevinsky, "Quasicommuting families of quantum Plücker coordinates", pp. 85-108 in Kirillov's seminar on representation theory, edited by G. I. Olshanski, Amer. Math. Soc. Transl. Ser. 2 181, Amer. Math. Soc., Providence, RI, 1998. MR 99g:14066 Zbl 0894.14021

[Lenagan and Rigal 2006] T. H. Lenagan and L. Rigal, "Quantum graded algebras with a straightening law and the AS-Cohen-Macaulay property for quantum determinantal rings and quantum Grassmannians", J. Algebra 301:2 (2006), 670-702. MR MR2236763

[Noumi et al. 1993] M. Noumi, H. Yamada, and K. Mimachi, "Finite-dimensional representations of the quantum group $\mathrm{GL}_{q}(n ; \mathbf{C})$ and the zonal spherical functions on $\mathrm{U}_{q}(n-1) \backslash \mathrm{U}_{q}(n)$ ", Japan. J. Math. (N.S.) 19:1 (1993), 31-80. MR 94i:17023 Zbl 0806.17016

[Parshall and Wang 1991] B. Parshall and J. P. Wang, "Quantum linear groups", Mem. Amer. Math. Soc. 89:439 (1991), vi+157. MR 91g:16028 Zbl 0724.17011

[Reshetikhin et al. 1989] N. Y. Reshetikhin, L. A. Takhtadzhyan, and L. D. Faddeev, "Quantization of Lie groups and Lie algebras", Algebra i Analiz 1:1 (1989), 178-206. MR 90j:17039 Zbl 0715.17015

[Scott 2005] J. Scott, "Quasi-commuting families of quantum minors", J. Algebra 290:1 (2005), 204-220. MR 2006d:16064 Zbl 1079.14058

[Soibelman 1990] Y. S. Solbel'man, "Algebra of functions on a compact quantum group and its representations", Algebra i Analiz 2:1 (1990), 190-212. In Russian; correction in Algebra i Analiz, 2:3 (1990), 256; translation in Leningrad Math. J. 2 (1991), 161-178. MR 91i:58053a

[Taft and Towber 1991] E. Taft and J. Towber, "Quantum deformation of flag schemes and Grassmann schemes, I: A q-deformation of the shape-algebra for GL(n)", J. Algebra 142:1 (1991), 1-36. MR 93e:17028 Zbl 0739.17007

Received February 15, 2005. Revised September 18, 2005.

\author{
KENNETH R. GOODEARL \\ DEPARTMENT OF MATHEMATICS \\ UNIVERSITY OF CALIFORNIA SANTA BARBARA \\ SANTA BARBARA, CA 93106 \\ UNITED STATES \\ goodearl@math.ucsb.edu
}

